\title{
Aharonov-Bohm ring with fluctuating flux
}

\author{
Florian Marquardt and C. Bruder \\ Department of Physics and Astronomy, University of Basel, Klingelbergstrasse 82, CH-4056 Basel, Switzerland
}

(Received 7 August 2001; revised manuscript received 19 November 2001; published 12 March 2002)

\begin{abstract}
We consider a noninteracting system of electrons on a clean one-channel Aharonov-Bohm ring that is threaded by a fluctuating magnetic flux. The flux derives from a Caldeira-Leggett bath of harmonic oscillators. We address the influence of the bath on the following properties: one- and two-particle Green's functions, dephasing, persistent current, and visibility of the Aharonov-Bohm effect in cotunneling transport through the ring. For the bath spectra considered here (including Nyquist noise of an external coil), we find no dephasing in the linear transport regime at zero temperature.
\end{abstract}

DOI: 10.1103/PhysRevB.65.125315

PACS number(s): 73.23.Hk, 73.23.Ra, 03.65.Yz

\section{INTRODUCTION}

In the present work, we consider a simple, theoretical model system of noninteracting spinless electrons that are restricted to move in one dimension around the circumference of a clean, one-channel Aharonov-Bohm ring. The ring is threaded by a magnetic flux that fluctuates around some average value (see Fig. 1). This may lead to dephasing of the electron motion on the ring, apart from other effects such as renormalization of the electron masses and introduction of an effective coupling between the electrons. We treat the full dynamics of the fluctuating flux coupled to the electron system in a self-consistent manner, rather than prescribing an external stochastic time-dependent classical field. In order to achieve this, the flux is taken to be the sum of the normal coordinates of a Caldeira-Leggett-type bath of harmonic oscillators. ${ }^{1,2}$ The fluctuations couple to the electrons via the vector-potential term in the kinetic energy. As an important special case for the bath spectrum we treat the Nyquist noise that may be due to the equilibrium current fluctuations in the external coil producing the flux.

Equilibrium and transport properties of this model system are analyzed for the cases of zero and finite temperatures, taking into account the coupling to the bath and the Pauli principle with respect to the electron system. In particular, we discuss the single-particle and two-particle Green's functions, level widths, energy shifts, and dephasing times, and the reduction of the persistent current due to the fluctuations. In each case, the dependence on the coupling strength between system and bath and on the low-frequency spectral properties of the bath is examined. Aharonov-Bohm interference observed in cotunneling through the ring is discussed in order to analyze the coherence properties of the electrons on the ring under the influence of the fluctuating flux in a transport situation. As a result of our calculation, we find that the fluctuations do not lead to dephasing in the linear transport regime at zero temperature.

The single-particle version of this model has been considered before in Ref. 3 in order to determine whether persistent currents in a normal metal ring may be destroyed by coupling to an Ohmic bath. Whereas the authors of Ref. 3 used the Feynman-Vernon influence functional, ${ }^{4}$ we will apply a different and more direct approach. We emphasize that our analysis is restricted to baths weaker than the Ohmic bath [see the discussion after Eq. (58)]. The possibility of spontaneous persistent currents was investigated (and ruled out) in Ref. 5, using a Luttinger liquid picture for the electrons and taking into account their electromagnetic self-interaction. Dephasing of a single electron going around the two arms of an Aharonov-Bohm ring has been considered both in Ref. 6, using the influence functional, and in Ref. 7, using a semiclassical picture. In the latter paper, the connection between phase fluctuations and the trace left by the system in the environment was emphasized. More recently, the question of dephasing in mesoscopic systems has received renewed attention due to a set of weak-localization measurements that have shown a saturation of the dephasing time at low temperatures. ${ }^{8,9}$ Motivated in part by these puzzling findings, the authors of Refs. 10-12 considered a ring containing a single quantum dot with fluctuating gate voltage and obtained the properties of the quantum-mechanical ground state (in particular the persistent current). A strong influence of external nonequilibrium noise on the persistent current in a disordered quasi one-dimensional ring has been found recently in Ref. 13. The effects of a phase-breaking scatterer in the many-particle situation, where the Pauli principle becomes important, have been discussed in Ref. 14. Very recently, dephasing in a mesoscopic Mach-Zehnder-type interference setup has been analyzed in Ref. 15.

In the following section, we will define the model and discuss some simple consequences as well as some features that cannot be included in this system easily. Then we give a short qualitative discussion of dephasing for the simplified case of a classical fluctuating flux (represented by a random process). Similar considerations are applied to the calcula-

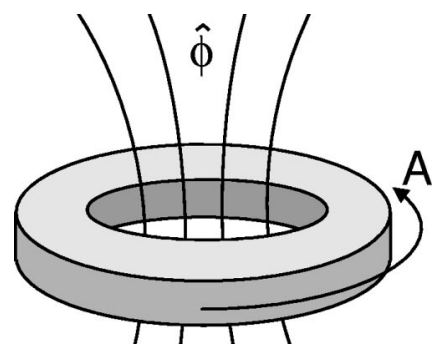

FIG. 1. The model situation. A fluctuating flux leads, via a timedependent vector potential, to a fluctuating force for the electrons on the Aharonov-Bohm ring. 
tion of the Green's function for a single electron on the ring, both with classical and quantum fluctuations. The resulting energy shifts and level shapes are analyzed in some detail, since these results can be taken over to the many-particle calculation of the single-particle and two-particle Green's functions that is presented in Sec. IV together with the evaluation of the grand canonical partition sum and the persistent current. After discussing the physical meaning of the dephasing produced by the Nyquist noise at low temperatures, we turn to an analysis of Aharonov-Bohm interference in a cotunneling transport measurement.

\section{THE MODEL}

The Hamiltonian of the system of electrons on the ring is given by

$$
\hat{H} \equiv \sum_{p} \hat{\Psi}_{p}^{\dagger} \frac{(p-g \hat{\phi})^{2}}{2 m} \hat{\Psi}_{p}+\hat{H}_{b a t h}
$$

where $\hat{H}_{\text {bath }}$ is the Hamiltonian of the set of uncoupled oscillators representing the bath

$$
\hat{H}_{\text {bath }} \equiv \sum_{j=1}^{N_{\text {osc }}}\left\{\frac{\hat{P}_{j}^{2}}{2 M}+\frac{M \omega_{j}^{2}}{2} \hat{Q}_{j}^{2}\right\} .
$$

The possible values of the electron momentum $p$ are quantized due to the finite circumference $L$ of the ring, $p$ $=2 \pi n / L$ with an integer $n$. (Note that here and in the following, we will put $\hbar \equiv k_{B} \equiv 1$ ) The term $g \hat{\phi}$ in the kinetic energy of the electrons is due to the coupling to the vector potential that is proportional to the fluctuating flux. $\hat{\phi}$ represents this flux (up to a constant factor) and is assumed to be given by the sum over the oscillator normal coordinates,

$$
\hat{\phi} \equiv \frac{1}{\sqrt{N_{\text {osc }}}} \sum_{j} \hat{Q}_{j}
$$

The prefactor in this definition has been chosen such that the autocorrelation function $\langle\hat{\phi}(t) \hat{\phi}(0)\rangle$ of $\hat{\phi}$ has a welldefined limit if the number $N_{\text {osc }}$ of oscillators tends to infinity while the spacing of frequencies tends to zero as $1 / N_{o s c}$. This is the "thermodynamic limit" of an infinite bath that is necessary to describe truly irreversible, dissipative dynamics. The quantity $g$ is the coupling strength between bath and electrons. It incorporates the electron charge and the circumference of the ring, since the line integral of the vector potential around the ring gives the flux. Any external static magnetic flux $\Phi$ has to be added in the kinetic-energy expression.

We assume the interaction between system and bath to be sufficiently weak, such that the bath may be treated as linear in a good approximation (as it is usually done in the theory of quantum dissipative systems, see Ref. 1 for a more detailed discussion). Apart from this assumption, the expression Eq. (3) used for the fluctuating flux is still completely general. We are free to choose the frequencies of the bath oscillators to obtain any desired correlation function of $\hat{\phi}$, which is the only quantity that affects the dissipative-system dynamics in this model. Note as well that the coupling of the velocity to a vector potential assumed in Eq. (1) has been shown ${ }^{16}$ to be equivalent to a Caldeira-Leggett model with the usual coordinate-type coupling for a particle moving on an infinite line. Physically, the coupling used here is the natural choice for a situation in which the vector potential is linearly related to the fluctuating current in an external coil. The fluctuations (and linear response) of the current then determine the bath correlator discussed in the following section.

The correlator of $\hat{\phi}$ will determine the dephasing rate and other important quantities via its low-frequency properties. For the discrete set of oscillators and for the continuum limit, respectively, it is given by the following expressions, where the average $\langle\cdot\rangle$ is taken with respect to the unperturbed set of oscillators,

$$
\begin{aligned}
\langle\hat{\phi}(t) \hat{\phi}(0)\rangle= & \frac{1}{N_{\text {osc }}} \sum_{j} \frac{1}{2 M \omega_{j}}\left[\operatorname{coth}\left(\frac{\omega_{j}}{2 T}\right) \cos \left(\omega_{j} t\right)\right. \\
& \left.-i \sin \left(\omega_{j} t\right)\right] \\
= & \int_{0}^{\infty} d \omega C(\omega)\left[\operatorname{coth}\left(\frac{\omega}{2 T}\right) \cos (\omega t)-i \sin (\omega t)\right] .
\end{aligned}
$$

This defines the spectral function $C(\omega)$ that we will use to characterize the bath spectrum. In terms of the discrete set of frequencies, it is given by $C(\omega) \equiv N_{o s c}^{-1} \Sigma_{j} \delta\left(\omega-\omega_{j}\right)$ $\times\left(2 M \omega_{j}\right)^{-1}$. Note that the coth is equal to $2 n(\omega)+1$, where $n(\omega)$ is the Bose-Einstein distribution function. The special case of Nyquist noise is obtained by the requirement that for high temperatures, the spectrum of fluctuations of the magnetic flux (i.e., of $\hat{\phi}$ ) is white. Since $\operatorname{coth}(\omega / 2 T)$ $=2 T / \omega$ for $T \gg \omega$, this means $C(\omega) \propto \omega$ (for small $\omega$ ).

Before we proceed to the calculations, we will point out some simplifying features of this situation as well as some important aspects of the dephasing problem in degenerate Fermion systems that are beyond the scope of this model.

The magnetic flux is assumed to thread the ring in such a way that the situation is axially symmetric with respect to the axis that goes through the center of the ring and is perpendicular to its plane. In this case, we can choose the gauge such that the vector potential is everywhere tangential to the ring and of constant magnitude around the whole circumference. The same holds for the electric field, which is given by the time derivative of the vector potential. This is analogous to the Caldeira-Leggett treatment of one-dimensional quantum Brownian motion of a free particle, with the formal difference that in our case the force is derived from a vector potential instead of a scalar potential. ${ }^{3,16}$ It is the choice appropriate for a system with periodic boundary conditions, where the quantization of momenta, the Aharonov-Bohm effect and persistent currents play a role. Note that under different circumstances the assumption of a force that is constant in space is only valid within the dipole approximation 
for a particle that is restricted to move in a well-localized region of space, because otherwise the finite wavelength of the bath modes (phonons etc.) becomes important.

Since the coupling between system and bath is via the momentum, which commutes with the electron Hamiltonian and therefore is a constant of the motion for the original uncoupled electron system ("diagonal coupling"), some features of this model are very simple. In spite of the interaction with the bath, the momentum of a particle will stay constant, only its velocity and kinetic energy can fluctuate. This simplifies the many-electron problem as well. Although the fluctuating force influences the center-of-mass motion of the electrons and introduces some kind of "effective interaction" between them, the occupation of different $p$ states cannot be changed by the bath. Note that this simplification would be spoiled if one takes into account impurities and/or a coupling that depends on the position. For example, the latter would arise if one considered an arbitrary fluctuating electromagnetic field or the electric field between the plates of a capacitor, which is a constant vector field in space but is not constant with respect to its projection onto the direction of motion of the electrons on the ring. Other situations where the coupling depends on position include interaction with phonons or a localized spin on the ring.

In its single-particle version, the Hamiltonian given above also arises in the discussion of dephasing for a charged island, if the coupling to the bath (e.g., a fluctuating gate voltage) is purely diagonal in the system's eigenbasis and can only lead to fluctuations in the energy levels. Note, however, that questions of interest here such as tunneling into the ring or features of the many-particle system have no natural counterpart in that rather simple situation.

Although in our model all electrons are coupled to the same flux, which introduces a kind of effective interaction between them, the decay rates of Green's functions will not show any dependence on the distance to the Fermi surface, in contrast to the usual behavior of interacting Fermi systems. This is due to the diagonal coupling between system and bath, which means that there are no energy-relaxation processes that change the occupation numbers of the electrons and that would feel the restriction by the Pauli principle. A related question arises in the study of dephasing in degenerate Fermion systems. If the coupling is not diagonal in the electrons' (single-particle) eigenstates, the system variable that couples to the bath (in our case the momentum) carries out fluctuations itself. In a semiclassical single-particle calculation, these fluctuations pick out the high-frequency components of the bath spectrum. ${ }^{9}$ Therefore, according to such a calculation, there is dephasing even in the case of a bath spectrum that vanishes at low frequencies and this implies that at low temperatures, the high-frequency zero-point fluctuations of the bath contribute heavily to dephasing in this picture. However, if the electron system is nearly degenerate, many of its transitions will be blocked by the Pauli principle so that such an effect will be strongly suppressed. Although we cannot investigate this point in our model, related considerations will occur in our discussion of the cotunneling setup in Sec. V.

\section{SINGLE-PARTICLE PROBLEM}

In this section, we will discuss the problem of a single particle on the ring, both semiclassically and quantum mechanically. The results can support the understanding of the following section, which is devoted to the many-particle situation.

\section{A. Semiclassical analysis}

Consider two wave packets traversing the left and right arm of the Aharonov-Bohm ring with constant velocity $v$ and meeting again after some time $t=L /(2 v)$ at the opposite end. The resulting interference pattern depends on the total phase difference between the two paths. In a semiclassical calculation, the phase difference is produced by the $v A$ term in the Lagrangian of the particle, and it is given by

$$
\varphi=2 \frac{e}{c} \int_{0}^{t} v A\left(t^{\prime}\right) d t^{\prime}
$$

The factor 2 arises because the phases are equal up to a change in sign. $A\left(t^{\prime}\right)$ gives the time dependence of the fluctuating vector potential that is assumed to be a classical Gaussian random process with zero mean in the hightemperature limit considered here. The visibility of the interference pattern will be suppressed due to the fluctuations in the phase $\varphi$. Since $\varphi$ is a Gaussian random variable, we obtain for the suppression factor

$$
\left\langle e^{i \varphi}\right\rangle=e^{-\left\langle\varphi^{2}\right\rangle / 2} .
$$

In our model $e A / c$ is equal to $g \phi$, where we will treat $\phi$ as a classical fluctuating field by taking into account only the real (symmetric) part of the correlator (4) in the hightemperature limit. Then the variance $\left\langle\varphi^{2}\right\rangle$ of the phase becomes

$$
\left\langle\varphi^{2}\right\rangle=4 g^{2} v^{2} \int_{0}^{t} d t_{1} \int_{0}^{t} d t_{2}\left\langle\phi\left(t_{1}\right) \phi\left(t_{2}\right)\right\rangle
$$

If the traversal time $t$ is much larger than the correlation time of the fluctuations in $\phi$, we may apply the following standard approximation:

$$
\begin{aligned}
\left\langle\varphi^{2}\right\rangle & \approx t 4 g^{2} v^{2} \int_{-\infty}^{+\infty} d t^{\prime}\left\langle\phi\left(t^{\prime}\right) \phi(0)\right\rangle \\
& =t 4 g^{2} v^{2} 4 \pi T\left(\frac{C(\omega)}{\omega}\right)_{\omega \rightarrow 0} .
\end{aligned}
$$

This means that in the case of Nyquist noise $(C \propto \omega)$ we obtain a finite "dephasing rate" that grows linearly with temperature. Note, however, that the time $t$ introduced here cannot grow without bounds but is fixed by the circumference $L$ and the velocity $v$. This calculation already shows that the dephasing rate will vanish for $v \rightarrow 0$ or $T \rightarrow 0$. For a bath that is weaker at low frequencies $\left(C \propto \omega^{\alpha}\right.$ with $\left.\alpha>1\right)$ we do not obtain a suppression factor that decays exponentially with time, hence the dephasing rate is always zero. We defer a 
more detailed discussion of the various cases to the full quantum-mechanical treatment below.

The physical interpretation of this result is clear. The fluctuating electric field proportional to $\dot{A}$ leads to a fluctuating velocity proportional to $A$, so that the random shift in the interference pattern is $\Delta x \propto \int A\left(t^{\prime}\right) d t^{\prime}$. The interference pattern will be completely washed out once the spread in $\Delta x$ becomes comparable to the wavelength $\lambda \propto 1 / v$. This coincides with the criterion $\left\langle\varphi^{2}\right\rangle \approx 1$.

Very similar considerations arise in the calculation of the Green's function of a single electron on the ring. The retarded Green's function may be approximated semiclassically by averaging the amplitude for propagation of the electron under the influence of the fluctuating flux

$$
\begin{aligned}
i G_{p}^{R}(t) & =\theta(t)\left\langle\left\{\hat{\Psi}_{p}(t), \hat{\Psi}_{p}^{\dagger}(0)\right\}\right\rangle \\
& \approx\left\langle\exp \left[-i \int_{0}^{t\left(p-g \phi\left(t^{\prime}\right)\right)^{2}} \frac{}{2 m} d t^{\prime}\right]\right\rangle .
\end{aligned}
$$

The exponential contains a quadratic term $(g \phi)^{2}$ that does not represent a Gaussian random variable, so that formula (6) cannot be applied to perform the averaging. Here and in the following quantum-mechanical calculation, we will neglect this term, which does not couple to the momentum (cf. discussion in Sec. IV E). With this approximation, the Green's function is given by an expression that again involves the correlation function of $\phi$

$$
\begin{aligned}
i G_{p}^{R}(t) \approx & \exp \left[-i \frac{p^{2}}{2 m} t\right] \\
& \times \exp \left[-\frac{1}{2}\left(\frac{g p}{m}\right)^{2} \int_{0}^{t} d t_{1} \int_{0}^{t} d t_{2}\left\langle\phi\left(t_{1}\right) \phi\left(t_{2}\right)\right\rangle\right] .
\end{aligned}
$$

At this point, the discussion given above applies. In particular, for Nyquist noise and finite temperatures, the Green's function decays exponentially with a rate proportional to $g^{2} p^{2} T$.

\section{B. Single-particle Green's function: Quantum case}

In the following, we will calculate and discuss the quantum-mechanical Green's function of a single electron on the ring. The extension to the many-particle case will be given in the following section. The single-particle density of states (DOS), which is given by the imaginary part of the Fourier transform of $G^{R}$, is a measurable quantity, as it can be revealed by tunneling into the ring.

We imagine a situation where the ring is empty and a single electron is inserted, so that the retarded Green's function is

$$
i G_{p}^{R}(t)=\theta(t)\left\langle\hat{\Psi}_{p t} \hat{\Psi}_{p 0}^{\dagger}\right\rangle=\theta(t)\left\langle e^{i \hat{H} t} \hat{\Psi}_{p} e^{-i \hat{H} t} \hat{\Psi}_{p}^{\dagger}\right\rangle .
$$

The average is a thermal expectation value with respect to the unperturbed bath of oscillators, corresponding to the situation without any particle on the ring. Obviously the bath cannot change the occupation of the different momentum states. Therefore, we only have to take into account that the time evolution between creation and destruction of the electron is governed by the Hamiltonian that contains the kineticenergy term that couples to the bath via the flux $\hat{\phi}$. Through this coupling, the introduction of the particle into the ring perturbs the bath oscillators. In anticipation of the manyelectron case, we will denote by $\hat{H}\left[\left\{p_{j}\right\}\right]$ the Hamiltonian for a fixed number of occupied momentum states, given by the set $\left\{p_{j}\right\}$. This operator only acts on the bath. Then the matrix element of the time-evolution operator with respect to the electron system is given by the following expression, for a Slater determinant belonging to the configuration $\left\{p_{j}\right\}$,

$$
\left\langle\left\{p_{j}\right\}\left|e^{-i \hat{H} t}\right|\left\{p_{j}\right\}\right\rangle=\exp \left(-i \hat{H}\left[\left\{p_{j}\right\}\right] t\right) .
$$

In particular, without any electrons we have $\hat{H}[0] \equiv \hat{H}_{b a t h}$. For the Green's function considered here, this leads to

$$
\begin{aligned}
\left\langle\hat{\Psi}_{p t} \hat{\Psi}_{p 0}^{\dagger}\right\rangle & =\left\langle e^{i \hat{H}[0] t} \exp (-i \hat{H}[\{p\}] t)\right\rangle \\
& =\left\langle\hat{T} \exp \left[-i \frac{p^{2}}{2 m} t+i \frac{g p}{m} \int_{0}^{t} d t^{\prime} \hat{\phi}\left(t^{\prime}\right)\right]\right\rangle .
\end{aligned}
$$

This expectation value can be interpreted as the (thermally averaged) overlap between the initial bath state evolved once with and once without presence of a particle on the ring. In the second line, we have introduced the interaction picture with respect to $\hat{H}_{b a t h}$ and dropped the term quadratic in $g \hat{\phi}$ from the kinetic energy (compare the discussion above). Since $\hat{\phi}$ is a bosonic variable (i.e., linear in the oscillator normal coordinates), Wick's theorem can be applied to the evaluation of this time-ordered thermal average, using a linked-cluster expansion. It leads to an expression completely analogous to the one used above for the classical Gaussian random process, see Eqs. (6) and (10). The difference consists in the replacement of the classical correlator by the thermal time-ordered expectation value:

$$
\begin{aligned}
& \left\langle\hat{T} \exp \left[i \kappa \int_{0}^{t} d t^{\prime} \hat{\phi}\left(t^{\prime}\right)\right]\right\rangle \\
& \quad=\exp \left[-\frac{\kappa^{2}}{2} \int_{0}^{t} d t_{1} \int_{0}^{t} d t_{2}\left\langle\hat{T} \hat{\phi}\left(t_{1}\right) \hat{\phi}\left(t_{2}\right)\right\rangle\right] .
\end{aligned}
$$

At present, $\kappa=g p / m$, but the same formula will be used below with other values for $\kappa$. In contrast to the classical correlator, $\left\langle\hat{T} \hat{\phi}\left(t_{1}\right) \hat{\phi}\left(t_{2}\right)\right\rangle$ is complex and will lead to an energy shift in addition to a decay of the Green's function. Using

$$
\begin{aligned}
\langle\hat{T} \hat{\phi}(t) \hat{\phi}(0)\rangle= & \int_{0}^{\infty} d \omega C(\omega)[\{2 n(\omega)+1\} \cos (\omega t) \\
& -i \sin (\omega|t|)]
\end{aligned}
$$

to evaluate the double time integral, we obtain for the exponent of Eq. (14), 

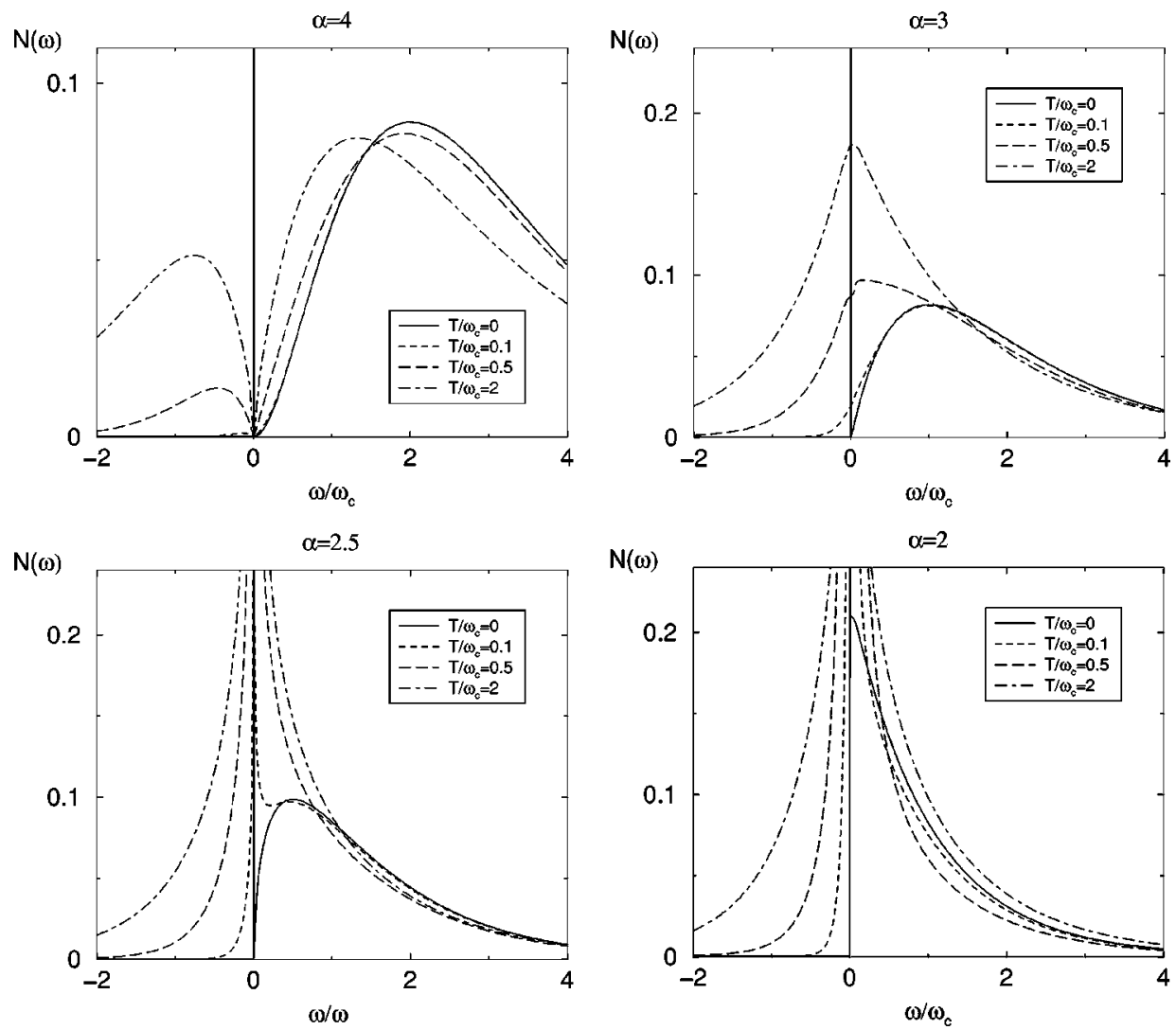

FIG. 2. The density of states for different bath spectra and temperatures. The quantity $N(\omega)$ $\equiv \operatorname{Im} K(\omega) / \pi$, which corresponds to the single-particle DOS obtained from the retarded Green's function, is plotted vs frequency $\omega / \omega_{c}$, for different exponents $\alpha$ of the bath spectrum at low frequencies $\left[C(\omega) \propto \omega^{\alpha} \exp \left(-\omega / \omega_{c}\right)\right]$ and for different temperatures. At $T=0$, the DOS goes as $\omega^{\alpha-2}$ at low $\omega$ and vanishes for $\omega<0$. In all cases displayed here, there is a $\delta$ peak at $\omega=0$, except for $\alpha=2$ at $T>0$. In the limit $T \rightarrow \infty$ the level shape becomes symmetric in all cases and the strength of the $\delta$ peak vanishes.

$$
\begin{aligned}
& i t \kappa^{2} \int_{0}^{\infty} d \omega \frac{C(\omega)}{\omega}+\kappa^{2} \int_{-\infty}^{+\infty} d \omega \frac{C(|\omega|)}{\omega^{2}}[n(|\omega|)+\theta(\omega)] \\
& \quad \times\left(e^{-i \omega t}-1\right) .
\end{aligned}
$$

The step function $\theta(\omega)$ corresponds to the zero-point fluctuations. In the remainder of this section, we will discuss the behavior of the Green's function derived from Eq. (16) at short and long times, for different bath spectra.

At short times, the integral in the second line compensates the first integral, so that the exponent begins to grow as $t^{2}$ instead of $t$. Physically, this means that at short times the particle has not yet influenced the bath and its energy is still given by the bare energy $p^{2} / 2 m$. At later times, the bath oscillators have been shifted by the presence of the particle, so the overlap between initial and final bath state is diminished and the Green's function decays. The first integral produces a negative energy shift, corresponding to the formation of an interacting state of particle and bath. Since this shift is proportional to $p^{2}$, it is equivalent to an enhanced effective mass $m^{*}$.

Combining the energy shift with the initial kinetic energy $p^{2} / 2 m$, we obtain the following expression for $m^{*}$,

$$
\frac{1}{m^{*}}=\frac{1}{m}\left(1-2 \frac{g^{2}}{m} \int_{0}^{\infty} d \omega \frac{C(\omega)}{\omega}\right) \equiv \frac{1}{m}(1-\xi) .
$$

Alternatively, the effective mass can be calculated from the initial Hamiltonian (for a single particle) by using the term $g p \hat{\phi} / m$ from the particle's kinetic energy to introduce a momentum-dependent shift into the oscillator potential energies of Eq. (2), $\hat{Q}_{j} \mapsto \hat{Q}_{j}-g p\left(m M \omega_{j}^{2} \sqrt{N_{o s c}}\right)^{-1}$. The resulting term quadratic in $p$ must be compensated for, which yields the change in mass,

$$
\frac{1}{m^{*}}=\frac{1}{m}\left(1-\frac{g^{2}}{m M} \frac{1}{N_{o s c}} \sum \frac{1}{\omega_{j}^{2}}\right) .
$$

Since we have neglected the term $(g \hat{\phi})^{2}$ in the preceding derivation, the effective mass displayed here is only correct in lowest order with respect to $g^{2}$. A full calculation yields $m^{*}=m(1+\xi)$.

The real part of the second integral in Eq. (16) gives a negative contribution, corresponding to a suppression in magnitude of the Green's function. If the bath is relatively weak at low frequencies ( $C \propto \omega^{\alpha}$ with $\alpha \geqslant 3$ ), the long-time behavior is simple. The decay saturates at $t \rightarrow \infty$.

In this case, the Fourier transform of the Green's function,

$$
G_{p}^{R}(\omega)=\int_{0}^{\infty} d t e^{i \omega t} G_{p}^{R}(t),
$$

still has a "quasiparticle" $\delta$ peak in the density of states $\operatorname{Im} G_{p}^{R}(\omega) / \pi$, but of a reduced magnitude. It is superimposed on an "incoherent background" (see Fig. 2).

For later use, we define the Fourier transform of the Green's function (without the constant energy shift) 


$$
\begin{aligned}
i K(\omega ; \kappa) \equiv & \int_{0}^{\infty} d t e^{i \omega t} \exp \left[\kappa^{2} \int_{-\infty}^{+\infty} d \tilde{\omega} \frac{C(|\tilde{\omega}|)}{\tilde{\omega}^{2}}\right. \\
& \left.\times[n(|\tilde{\omega}|)+\theta(\tilde{\omega})]\left(e^{-i \tilde{\omega} t}-1\right)\right] .
\end{aligned}
$$

For a bath with a spectrum that is stronger at low frequencies $(\alpha \leqslant 2)$, the Green's function may decay to zero for $t$ $\rightarrow \infty$, which means that there is no $\delta$ peak any more in the density of states.

The resulting single-particle density of states is presented in Fig. 2 for different values of the exponent $\alpha$ characterizing the strength of the bath spectrum at low frequencies, both for zero and finite temperatures.

Here, we will analyze in more detail the case of Nyquist noise, with a linear spectrum $C(\omega)=C_{0} \omega$ at small frequencies.

If the temperature is finite, we may set $n(\omega) \approx T / \omega$ for sufficiently low frequencies. The real part of the exponent (16) then becomes, in the long-time limit,

$$
-2 \pi C_{0} \kappa^{2} T t
$$

The prefactor in this expression defines the decay rate for the Green's function, which determines the width of the Lorentzian that arises in the density of states.

At zero temperature the thermal excitation of the bath oscillators vanishes, so only the zero-point contribution remains. In that case, we have to specify the behavior of the bath spectrum at high frequencies, because it becomes important even at $t \rightarrow \infty$. Choosing either a sharp cutoff $C(\omega)$ $=C_{0} \omega \theta\left(\omega_{c}-\omega\right)$ or an exponential decay $C(\omega)=C_{0} \omega$ $\times \exp \left(-\omega / \omega_{c}\right)$ results in the same long-time behavior of the relevant integral

$$
\int_{0}^{\infty} d \omega \frac{C(\omega)}{\omega^{2}}[\cos (\omega t)-1]=-C_{0} \ln \left(\omega_{c} t\right)+h(t)
$$

Here $h(t)$ is a function that saturates to a constant value for $t \rightarrow \infty$. The logarithm in the exponent leads to a powerlaw decay of the Green's function

$$
G_{p}^{R}(t) \propto\left(\omega_{c} t\right)^{-\gamma}
$$

with an exponent $\gamma$ proportional to the strength of the bath, the coupling and the momentum squared,

$$
\gamma=\kappa^{2} C_{0}=\left(\frac{g p}{m}\right)^{2} C_{0} .
$$

The detailed behavior of the line shape (not only the linewidth) then depends on $\gamma$, see Fig. 3.

We will encounter such a power-law decay again in the discussion of dephasing for the Nyquist bath. It is similar to the power-law behavior found in the model of quantum Brownian motion. ${ }^{1,17,18}$ However, there are important differences between the Nyquist bath considered here and the (stronger) Ohmic bath. These will be discussed in Sec. IV D.

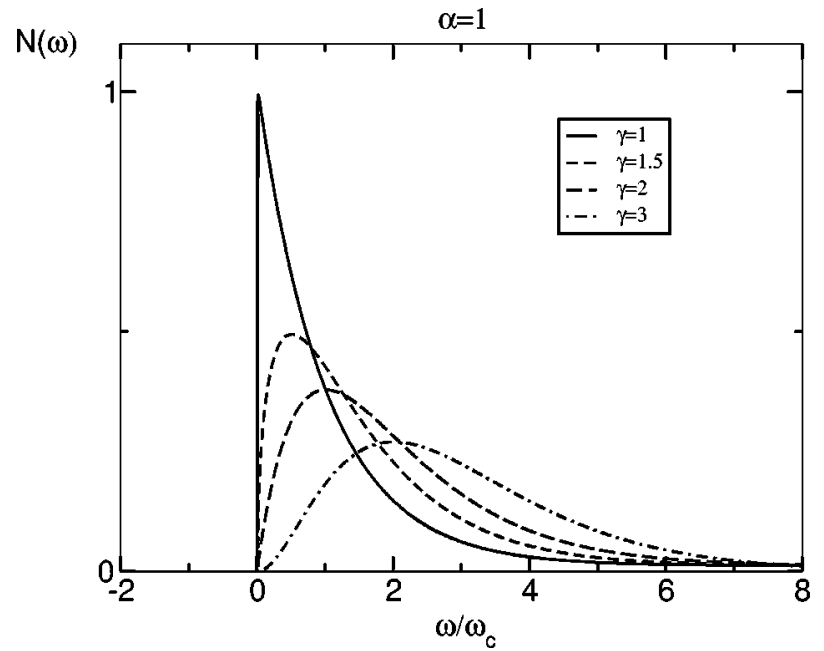

FIG. 3. The density of states for Nyquist noise $(\alpha=1)$ at $T$ $=0$, for different values of the power-law exponent $\gamma$. At finite temperature $T$, this goes over into a Lorentz peak of width proportional to $T$, see text.

\section{MANY-PARTICLE PROBLEM}

Now we consider the situation of many electrons on the ring, in the grand canonical ensemble at a given value of the chemical potential $\mu$ and at an arbitrary temperature $T$ $=\beta^{-1}$.

\section{A. Grand canonical partition sum and persistent current}

As a preparation for the evaluation of various averages carried out below, we need the grand canonical partition sum

$$
\begin{aligned}
Z_{g c}(\beta, \mu) & =\sum_{N=0}^{\infty} e^{\beta \mu N} \operatorname{tr}\left(e^{-\beta \hat{H}_{N}}\right) \\
& =\sum_{N} e^{\beta \mu N} \sum_{\left\{p_{j}\right\}} \operatorname{tr}_{B}\left(\exp \left(-\beta \hat{H}\left[\left\{p_{j}\right\}\right]\right)\right) .
\end{aligned}
$$

Here we have used the notation $\left\{p_{j}\right\}$ (that has been introduced above) for a given configuration of $N$ particles. $\operatorname{tr}_{B}$ in the second line denotes the trace with respect to the bath oscillators. If we introduce an additional static magnetic flux $\Phi$, this corresponds to a shift of the momenta $p_{j} \mapsto p_{j}$ $-e \Phi / L c$ in all the formulas given below, such that the partition sum becomes dependent on $\Phi$ as well. Note that we assume the effects of the charging energy of the ring to be negligible here, unlike the treatment of cotunneling in Sec. V.

The Hamiltonian $\hat{H}\left[\left\{p_{j}\right\}\right]$ can be split into a part containing only the momenta $p_{j}$ and another one that represents bath oscillators that are shifted (depending on $p_{j}$ ) but still have the original frequencies (if we neglect the $g^{2} \hat{\phi}^{2}$ term such as above),

$$
\sum_{j=1}^{N} \frac{p_{j}^{2}-2 g p \hat{\phi}}{2 m}+\hat{H}_{b a t h}=T\left[\left\{p_{j}\right\}\right]+\hat{H}_{b a t h}\left[\left\{p_{j}\right\}\right]
$$


where $\hat{H}_{b a t h}\left[\left\{p_{j}\right\}\right]$ is derived from the original bath Hamiltonian (2) by a shift in oscillator coordinates

$$
\hat{Q}_{l} \mapsto \hat{Q}_{l}-\frac{g}{m M \omega_{l}^{2} \sqrt{N_{\text {osc }}}} \sum_{j} p_{j},
$$

and $T\left[\left\{p_{j}\right\}\right]$ is the residual "kinetic-energy" term that only depends on the set of occupied momenta $\left\{p_{j}\right\}$,

$$
T\left[\left\{p_{j}\right\}\right] \equiv \frac{1}{2 m}\left[\sum_{j} p_{j}^{2}-\xi\left(\sum_{j} p_{j}\right)^{2}\right] .
$$

Here we have used the constant factor $\xi$ defined in the discussion of the effective mass, see Eq. (17). $T\left[\left\{p_{j}\right\}\right]$ differs from the single-particle case by the appearance of the total momentum $P=\Sigma p_{j}$, which makes it impossible to write this as a sum over the kinetic energies of individual electrons with renormalized masses. Physically, all the electrons are coupled to one and the same bath and this influences the center-of-mass motion. The dependence of $T\left[\left\{p_{j}\right\}\right]$ on the total momentum introduces a kind of simple "effective interaction" between the electrons, which affects thermodynamic averages. For example, the average occupation number for a given momentum does not follow the Fermi-Dirac distribution. Note that formally the kinetic energy $T\left[\left\{p_{j}\right\}\right]$ can become negative for large total momenta $P$ if $\xi>1 / N$. However, this only means that higher orders in $\xi$ would have to be taken into account (neglecting $g^{2} \hat{\phi}^{2}$ becomes invalid), as discussed already in connection with the effective mass for a single particle [see Eq. (17)].

Using this definition, we can rewrite $Z_{g c}$, taking into account that the partition sum of the bath of shifted harmonic oscillators is equal to that of the unperturbed bath $Z_{H O}$ and therefore does not depend on $\left\{p_{j}\right\}$,

$$
Z_{g c}=Z_{H O} \sum_{N} e^{\beta \mu N} \sum_{\left\{p_{j}\right\}} e^{-\beta T\left[\left\{p_{j}\right\}\right]} .
$$

The partition sum without the contribution due to the harmonic oscillators will be denoted by $\widetilde{Z}_{g c}$ from now on,

$$
\widetilde{Z}_{g c} \equiv \frac{Z_{g c}}{Z_{H O}} .
$$

We can simplify Eq. (29) further by rewriting $\widetilde{Z}_{g c}$ in the following way, using both the average $\langle\cdot\rangle_{0}$ and the partition sum $\widetilde{Z}_{g c}^{0}$ with respect to the system of free electrons

$$
\begin{aligned}
\widetilde{Z}_{g c} & =\sum_{N,\left\{p_{j}\right\}} \exp \left[-\beta \sum_{j}\left(p_{j}^{2} / 2 m-\mu\right)\right] e^{\beta \xi P^{2} / 2 m} \\
& =\widetilde{Z}_{g c}^{0}\left\langle e^{\beta \xi \hat{P}^{2} / 2 m}\right\rangle_{0} \approx \widetilde{Z}_{g c}^{0}\left(1+\beta \frac{\xi}{2 m}\left\langle\hat{P}^{2}\right\rangle_{0}\right) .
\end{aligned}
$$

Here, we have kept only the lowest nonvanishing order in $\xi$, since everything else would be inconsistent within the framework of our approximation.
Now we can apply these results to the calculation of the persistent current of our system of electrons coupled to the fluctuating flux.

The average current that flows for a given external magnetic flux $\Phi$ is the derivative of the thermodynamic potential $\Omega=-T \ln Z_{g c}$ with respect to the flux itself,

$$
\begin{aligned}
\langle\hat{I}\rangle & =c T \frac{\partial}{\partial \Phi} \ln Z_{g c} \\
& =c T \frac{\partial}{\partial \Phi}\left\{\ln \widetilde{Z}_{g c}^{0}+\beta \frac{\xi}{2 m}\left\langle(\hat{P}-\hat{N} e \Phi / c L)^{2}\right\rangle_{0}\right\} \\
& =\frac{e}{L}\langle\hat{V}(1-\xi \hat{N})\rangle_{0} .
\end{aligned}
$$

Here $\hat{V}=[\hat{P}-\hat{N} e \Phi /(c L)] / m$ is the total velocity operator for the electrons. We have replaced $\hat{P}$ in Eq. (31) by the expression valid in the presence of the external static flux. Obviously, the persistent current is reduced by a factor of about $(1-\xi N)$. The result does not depend on the details of the bath spectrum, only on $\xi$, which is well behaved also for the case of the Nyquist bath. Recall that for values $\xi>1 / N$ the approximation used here is invalid, as explained above.

The magnitude of the reduction of the persistent current may be understood physically in the following way. If one imagines suddenly switching on the external magnetic flux, an electric-field pulse will be produced, which, at first, freely accelerates the electrons on the ring, leading to a current that is proportional to the number $N$ of electrons. This again produces a change in the magnetic flux that prompts a reaction of the bath (e.g., the external coil producing the Nyquist noise). The back action onto the electrons deccelerates them, decreasing the velocity of each electron by an amount proportional to $N$ and depending on the coupling strength between the ring and the external coil, which is contained in $\xi$. This leads to the reduction factor $1-\xi N$ obtained above.

Thus, in our model, the reduction of the persistent current is similar in its origin to the appearance of an effective mass. This can be seen most clearly by considering the special case of a "fast" bath, whose spectrum has a lower frequency cutoff or, at least, vanishes quickly with decreasing frequency. It follows the motion of the electrons adiabatically and is not able to lead to dephasing on long time scales (see the discussion of the two-particle Green's function below, as well as the cotunneling setup discussed in the last section). However, it still leads to a reduction of the persistent current, since the quantity $\xi$ represents an integral over all frequencies.

\section{B. Single-particle Green's function}

The single-particle Green's function is defined by

$$
i G_{p}(t)=\left\langle\hat{\Psi}_{p t} \hat{\Psi}_{p 0}^{\dagger}\right\rangle \theta(t)-\left\langle\hat{\Psi}_{p 0}^{\dagger} \hat{\Psi}_{p t}\right\rangle \theta(-t) .
$$

Evaluation of both expectation values proceeds in the same way, so we will only treat the first one here 


$$
\left\langle\hat{\Psi}_{p t} \hat{\Psi}_{p 0}^{\dagger}\right\rangle=Z_{g c}^{-1} \sum_{N} e^{\beta \mu N} \operatorname{tr}\left(e^{-\beta \hat{H}_{N}} e^{i \hat{H}_{N} t} \hat{\Psi}_{p} e^{-i \hat{H}_{N} t} \hat{\Psi}_{p}^{\dagger}\right) .
$$

The trace is evaluated by summing over all configurations $\left\{p_{j}\right\}$ of $N$ particles and using the fact that the bath does not change a given configuration. Therefore it is equal to

$$
\begin{aligned}
\sum_{\left\{p_{j}\right\}}\left(1-n_{p}\left[\left\{p_{j}\right\}\right]\right) \operatorname{tr}_{B}\left(\exp \left(-\beta \hat{H}\left[\left\{p_{j}\right\}\right]\right) \exp \left(i \hat{H}\left[\left\{p_{j}\right\}\right] t\right)\right. \\
\left.\quad \times \exp \left(-i \hat{H}\left[\left\{p_{j}\right\}^{\prime}\right] t\right)\right) .
\end{aligned}
$$

Here $\left\{p_{j}\right\}^{\prime}$ is the configuration with one particle added in state $p$, and the prefactor is zero whenever that state is already occupied. Now we introduce the interaction picture with respect to $\hat{H}\left[\left\{p_{j}\right\}\right]$. Furthermore, we will use the partition sum for a given configuration,

$$
Z_{\left\{p_{j}\right\}}=\operatorname{tr}_{B}\left(\exp \left(-\beta \hat{H}\left[\left\{p_{j}\right\}\right]\right)\right)=Z_{H O} \exp \left(-\beta T\left[\left\{p_{j}\right\}\right]\right),
$$

in order to define the average over the bath oscillators that are shifted depending on $\left\{p_{j}\right\}$,

$$
\begin{aligned}
\langle\cdots\rangle_{\left\{p_{j}\right\}} & \equiv Z_{\left\{p_{j}\right\}}^{-1} \operatorname{tr}_{B}\left(\exp \left(-\beta \hat{H}\left[\left\{p_{j}\right\}\right]\right) \ldots\right) \\
& =Z_{H O}^{-1} \operatorname{tr}_{B}\left(\exp \left(-\beta \hat{H}_{b a t h}\left[\left\{p_{j}\right\}\right]\right) \ldots\right) .
\end{aligned}
$$

(See the discussion of $Z_{g c}$ in Sec. IV A for a definition of the quantities $Z_{H O}$ and $\hat{H}_{b a t h}\left[\left\{p_{j}\right\}\right]$.)

With these definitions, expression (35) becomes

$$
\begin{aligned}
\sum_{\left\{p_{j}\right\}} Z_{\left\{p_{j}\right\}}\left(1-n_{p}\left[\left\{p_{j}\right\}\right]\right) & \\
& \times\left\langle\hat{T} \exp \left[-i \frac{p^{2}}{2 m} t+i \frac{g p}{m} \int_{0}^{t} d t^{\prime} \hat{\phi}\left(t^{\prime}\right)\right]\right\rangle_{\left\{p_{j}\right\}} .
\end{aligned}
$$

In evaluating the average of the exponential, we need the expectation value of $\hat{\phi}$ that does not vanish in this case, since the oscillators are shifted by an amount proportional to the total momentum of the given configuration,

$$
g \bar{\phi}=g\langle\hat{\phi}\rangle_{\left\{p_{j}\right\}}=\xi\left(\sum_{j} p_{j}\right) .
$$

Apart from this, we can proceed exactly as before in the strictly single-particle case, see Eq. (14), in order to arrive at an exponent involving the thermal time-ordered correlator of $\hat{\phi}$ for the unperturbed harmonic oscillators. Using this and Eq. (39) to evaluate Eq. (38), we have arrived at the desired result for one half of the Green's function, Eq. (34). Proceeding analogously for the other half and using the definition (20) for $K(\omega ; \kappa)$, the Fourier transform of the Green's function is given by

$$
\begin{aligned}
G_{p}(\omega)= & \widetilde{Z}_{g c}^{-1} \sum_{\left\{p_{j}\right\}} \exp \left[-\beta\left\{\sum_{j}\left(\frac{p_{j}^{2}}{2 m}-\mu\right)-\frac{\xi}{2 m} P^{2}\right\}\right] \\
& \times\left\{\left(1-n_{p}\left[\left\{p_{j}\right\}\right]\right) K\left(\omega-\frac{p^{2}}{2 m^{*}}+\frac{\xi}{m} p P ; \frac{g p}{m}\right)\right. \\
& \left.-n_{p}\left[\left\{p_{j}\right\}\right] K\left[-\omega+\frac{p^{2}}{2 m}(1+\xi)-\frac{\xi}{m} p P ; \frac{g p}{m}\right]\right\} .
\end{aligned}
$$

The sum runs over all configurations (of any particle number $N$ ) and $P=\Sigma p_{j}$ is the total momentum.

From this result one can see that the line shape of the DOS in the many-particle case is derived from the singleparticle result $K(\omega ; \kappa)$. However, at finite temperatures, it is the average over many such curves, each shifted by an amount proportional to $p P$ that depends on the total momentum of the configuration. Still, the linewidth (or line shape) does not depend in any essential way on the distance to the Fermi surface.

We will now discuss the temperature dependence of the linewidth that results from expression (40). The $\delta$ peak that remains in $K(\omega)$ at $\omega=0$ for a "weak bath" (in this case, $\alpha>2$ ) is smeared over a certain range due to the average over configurations with different total momenta $P$. In lowest order with respect to $\xi$, we may neglect the dependence of the probability distribution on the coupling to the bath. Then, the linewidth is obviously given by

$$
\delta \omega=\frac{\xi}{m} p \delta P
$$

where $\delta P=\sqrt{\left\langle P^{2}\right\rangle_{0}}$ is the spread in total momentum, calculated for the original free-electron system. We have $\left\langle P^{2}\right\rangle_{0}$ $=N m T$ and therefore a linewidth which increases with the square root of $T$,

$$
\delta \omega \propto \xi \sqrt{T} .
$$

Note that the corresponding spread $\delta p=\delta \omega / v$ in momentum space is given by $\xi \sqrt{N m T}$ and can very well exceed the distance $2 \pi / L$ of the quantized momenta, in spite of the restriction $\xi N \ll 1$ (and also in spite of the restriction $\sqrt{m T}$ $\ll p_{F}$ for the degenerate regime). Therefore, it is reasonable to speak of a linewidth, provided one does not resolve the quantized level structure on the ring.

\section{Dephasing: Two-particle Green's function}

While the decay of the single-particle Green's function in time is connected with every interaction process that changes the state of the electron or brings about random changes in its phase, it is not sufficient to know about this decay if one asks about dephasing. After all, there are situations where an electron interacts with a bath, such that its Green's function decays quickly but it is still able to show an interference pattern. This will happen whenever the trace left by the particle in the bath is not enough to decide which path it has 
gone, so that the different possibilities still interfere. ${ }^{7}$ Therefore, one must ask about the time evolution of the density matrix (and, in particular, the decay of its off-diagonal elements) in order to study dephasing. Given a small initial perturbation that creates a nonequilibrium situation, this time evolution is determined by the two-particle Green's function in a linear response calculation.

The following calculation basically proceeds along the same lines as that given in the preceding section, so we will keep it brief. For our purposes, we do not need the twoparticle Green's function for arbitrary values of the four time arguments, but only for a perturbation acting at time zero and a density matrix evaluated at time $t$. Furthermore, since the bath does not change the occupation of momentum states, the only nontrivial contribution arises from the following product of four-electron operators, in which only two momenta $p$ and $p^{\prime}$ appear. $p$ refers to the hole that is created by the perturbation while $p^{\prime}$ belongs to the electron.

$$
\begin{aligned}
& \left\langle\hat{\Psi}_{p t}^{\dagger} \hat{\Psi}_{p^{\prime} t} \hat{\Psi}_{p^{\prime} 0}^{\dagger} \hat{\Psi}_{p 0}\right\rangle \\
& \quad=Z_{g c}^{-1} \sum_{N} e^{\beta \mu N} \operatorname{tr}\left(e^{-\beta \hat{H}_{N}} e^{i \hat{H}_{N} t} \hat{\Psi}_{p}^{\dagger} \hat{\Psi}_{p^{\prime}} e^{-i \hat{H}_{N} t} \hat{\Psi}_{p^{\prime}}^{\dagger} \hat{\Psi}_{p}\right) .
\end{aligned}
$$

Inserting an appropriate basis of system states $\left|\left\{p_{j}\right\}\right\rangle$, using the interaction picture with respect to $\hat{H}\left[\left\{p_{j}\right\}\right]$ and carrying out the average of the exponential in the usual way, we arrive at the following result for the (half-sided) Fourier transform of Eq. (43),

$$
\begin{aligned}
& \widetilde{Z}_{g c}^{-1} \sum_{\left\{p_{j}\right\}} \exp \left(-\beta\left\{\sum_{j}\left(\frac{p_{j}^{2}}{2 m}-\mu\right)-\frac{\xi}{2 m} P^{2}\right\}\right) n_{p}\left[\left\{p_{j}\right\}\right] \\
& \quad \times\left(1-n_{p^{\prime}}\left[\left\{p_{j}\right\}\right]\right) i K\left(\omega-\frac{p^{\prime 2}-p^{2}}{2 m}+\frac{\xi}{2 m}\left(p^{\prime}-p\right)^{2}\right. \\
& \left.\quad+\frac{\xi}{m}\left(p^{\prime}-p\right) P ; \frac{g\left(p^{\prime}-p\right)}{m}\right) .
\end{aligned}
$$

The notation is the same as for Eq. (40). The most important difference consists in replacing $p$ by $p^{\prime}-p$ in the factor $\kappa=g p / m$ that determines the strength of the decay.

For the Nyquist case, we thus obtain a finite dephasing rate

$$
\frac{1}{\tau_{\varphi}\left(p, p^{\prime}\right)}=2 \pi T C_{0}\left(\frac{g}{m}\right)^{2}\left(p^{\prime}-p\right)^{2},
$$

which is proportional to the difference in momenta squared, the bath, coupling strengths, and the temperature. The change in phase brought about by the fluctuating flux is proportional to $p$, so the phase difference, whose variance appears in the exponent, goes as $p^{\prime}-p$. To avoid confusion, we emphasize that there is no universally applicable definition of a "dephasing rate." In our case, we use this term to refer to the exponential decay of the two-particle Green's function as introduced above. Note that the dephasing rate can be small even if the decay rates associated with the (single- particle) Green's functions of the individual states are large. This is an example of the general behavior mentioned above. It is reasonable that dephasing is strongest whenever the momenta of the two states, whose superposition is examined, differ widely. Then, the bath, which couples to the momentum, can easily distinguish between these states even after a short time.

The dephasing rate $\tau_{\varphi}^{-1}$ vanishes at $T=0$. However, even at $T=0$ the off-diagonal element of a density matrix that initially describes a coherent superposition between momentum states $p$ and $p^{\prime}$ decays to zero completely in the limit $t \rightarrow \infty$. This decay proceeds with a power law, as we have already observed for the single-particle Green's function, see Eq. (23). In the situation considered here, the exponent $\gamma$ is equal to

$$
\gamma=\left(\frac{g}{m}\right)^{2}\left(p^{\prime}-p\right)^{2} C_{0}
$$

If the bath is sufficiently weak at low frequencies $\left[C(\omega)=C_{0} \omega^{\alpha}\right.$ with $\left.\alpha>2\right]$, the decay of the off-diagonal elements in time saturates at a finite value, in contrast to the Nyquist case discussed above. Then the dephasing rate, defined as the prefactor of $t$ in the exponential decay law, is strictly zero, even at finite temperatures. This behavior is related to the diagonal coupling between system and bath. If a nondiagonal coupling were introduced, there would be transitions from excited electronic states towards lower ones, accompanied by the spontaneous emission of a bath phonon. In that case, the decay rate in the single-particle Green's function and the dephasing rate would be nonzero also at $T$ $=0$ but strongly dependent on the distance to the Fermi surface, due to the suppression of the density of final states for such transitions brought about by the Pauli principle. Therefore, in a simple Golden-rule calculation, these decay rates would vanish at $T=0$ when one approaches the Fermi surface.

For $\alpha=2$, we find power-law dephasing only at finite temperatures, with an exponent proportional to $T$.

\section{Discussion of dephasing for the "Nyquist bath"}

The Nyquist bath is characterized by a fluctuation spectrum of flux and vector potential that is linear in $\omega$ (at zero temperature), therefore leading to a spectrum for the electric field that behaves as $\omega^{3}$. This is exactly the spectrum of the zero-point fluctuations of the electric field in the vacuum. The main distinction between those fluctuations and the Nyquist noise considered here is that the latter leads to a force that is homogeneous around the ring and therefore is compatible with the translational invariance of our onedimensional system of electrons. Furthermore, its magnitude depends on the geometry and resistance of the external circuit producing the equilibrium current noise. Apart from these differences, we can use our understanding of the electromagnetic vacuum fluctuations to discuss the effects of the Nyquist bath in a qualitative manner.

In particular, free ballistic motion is not affected, since the radiation reaction force only acts on an accelerated charge. 
Therefore, the populations of the electronic momentum eigenstates do not decay, as we have already observed. This is in contrast to the effect of a type of bath that leads to velocity-proportional friction, for example. The system is not ergodic, since the memory of the initial conditions is not lost completely. In a basis other than the momentum basis, the off-diagonal elements of the density matrix show only partial decay. That there is some decay of the coherences is connected to the smearing of the position of the particle in the course of time. Usually, this effect is neglected in the discussion of dissipative quantum motion of a free particle under the influence of a bath corresponding to electromagnetic vacuum fluctuations, since the ballistic expansion of an initially localized ensemble of particles dominates. ${ }^{18}$ In our case, it is important, since, for example, a superposition of two counterpropagating plane waves on the ring will first form a standing wave pattern, whose visibility then gradually decreases. The fact that, at $T=0$, the decay of the visibility proceeds as a power law can be understood most easily from the results of an old semiclassical analysis of the Lamb shift due to Welton. ${ }^{19,20}$ The vacuum fluctuations of the electric field lead to a jitter of the electron position, such that the variance $\left\langle\delta x^{2}\right\rangle$ of its coordinate is given by the logarithm containing the ratio of an upper cutoff frequency (there taken to be the Compton frequency) and a lower cutoff (the characteristic frequency of electron motion around the nucleus). In our case, the lower cutoff frequency actually is given by the inverse of the observation time, such that $\left\langle\delta x^{2}\right\rangle$ $\propto \ln \left(\omega_{c} t\right)$. For a superposition of plane waves of momenta $\pm k$ on the ring, the density matrix in position space contains cross terms such as $\exp (i 2 k x)$, which, if averaged over $\delta x$, give rise to a suppression factor $\exp \left(-2 k^{2}\left\langle\delta x^{2}\right\rangle\right) \propto t^{-\gamma}$. This leads to complete decay of the interference pattern even at $T=0$. Note, however, that here we have been considering a superposition of excited states of the system and the decay of its coherences. In other problems of dephasing, such as those encountered in weak localization, one usually discusses the limit of zero-frequency response of the system to a small perturbation. A situation that comes closer to this kind of question will be discussed in the following section.

The goal of the present work has been to analyze thoroughly a model situation that shows some of the features important for dephasing at low temperatures, not to propose some experimental measurement setup. Still, we will now briefly discuss the expected magnitude of the effect due to Nyquist noise in an external current coil. If the equilibrium current fluctuations are produced by an external coil whose circumference is similar to that of the Aharonov-Bohm ring $(L)$ and that is placed about a distance $L$ away, the dephasing rate is estimated to be

$$
\frac{\hbar}{\tau_{\varphi}} \sim\left(\frac{e^{2}}{\hbar c}\right)^{2}\left(\frac{v_{F}}{c}\right)^{2} \frac{k_{B} T}{R / R_{K}} .
$$

Here $v_{F}$ is the Fermi velocity on the ring, $R$ is the resistance of the external coil, and $R_{K}=h / e^{2}$ is the quantum of resistance. Both the square of the fine-structure constant in front of the expression and the ratio of the Fermi velocity to the speed of light render the effect very small under reasonable experimental conditions.

Note that the fluctuations of the vacuum magnetic field have a weaker power spectrum, which leads to $\langle A A\rangle_{\omega} \propto \omega^{3}$ at $T=0$, instead of $\langle A A\rangle_{\omega} \propto \omega$. The vacuum fluctuations of the electric field, however, do lead to a linear spectrum in the vector-potential fluctuations (see discussion above). On the other hand, the electric field (at large wavelengths) is homogeneous only in free space, not with respect to its projection onto the ring, where it has a position dependence $\cos (2 \pi x / L)$. Thus, the coupling is not diagonal in the momentum basis and is not included in our model. If one estimates the order of magnitude of the corresponding dephasing rate (inelastic transition rate), one arrives at $\tau_{\varphi(e m)}^{-1} \sim\left(e^{2} / \hbar c\right)$ $\times\left(v_{F} / c\right)^{2}\left(v_{F} / L\right)$. Similarly, one may estimate the strength of fluctuations due to shot noise of the external current. In a situation where the external coil producing a static magnetic flux on the order of $\sim \Phi_{0}$ is identical with that where the Nyquist noise originates, this leads to an effective dephasing rate of $\tau_{\varphi(s h o t)}^{-1} \sim\left(e^{2} / \hbar c\right)\left(v_{F} / c\right) v_{F} / L$, which may be much larger than that due to the Nyquist noise. Note that shot noise cannot be described by our model, since it is a nonequilibrium phenomenon and cannot be represented by the usual bath of harmonic oscillators at low temperatures. However, the effects of shot noise would be reduced in a different geometry where a larger current (with correspondingly smaller relative magnitude of the shot noise) produces the same static magnetic flux through the Aharonov-Bohm ring.

\section{E. Relevance of the term quadratic in the flux}

In all of the preceding calculations, we have neglected the term $g^{2} \hat{\phi}^{2}$ that appears in the kinetic energy of the particle but does not couple to the momentum. This approximation has been necessary to use the well-known formula $\langle\exp (i X)\rangle=\exp \left(-\left\langle X^{2}\right\rangle / 2\right)$ for a Gaussian random variable $X$ in the classical case or the analogous expression derived from Wick's theorem in the quantum case. $\hat{\phi}^{2}$ is quadratic in the coordinates of the bath oscillators, whereas $\hat{\phi}$ is a linear bosonic variable (Gaussian random variable in the classical case). If the term $g^{2} \hat{\phi}^{2} / 2 m$ is kept in the Hamiltonian, the eigenfrequencies and normal coordinates of the bath of harmonic oscillators are changed by an amount that depends on $g$ but not on the particle momentum $p$. This becomes important at larger values of $g$, where the effective mass turns out to be $m^{*}=m(1+\xi)$ instead of the value $m^{*}=m(1-\xi)^{-1}$ derived without the $\hat{\phi}^{2}$ term, see Eq. (17). Note that this difference persists also in the thermodynamic limit $N_{\text {osc }}$ $\rightarrow \infty$. However, the qualitative behavior of the bath spectrum at low frequencies is not changed for the spectra $C(\omega)$ $\propto \omega^{\alpha}$ with $\alpha \geqslant 1$ that have been considered here. Therefore, this term is unimportant for the qualitative conclusions about dephasing, although it can change quantitative results for larger $g$ and does change the single-particle Green's function. The latter involves a change in particle number, so that an additional $\hat{\phi}^{2}$ is introduced into the Hamiltonian. 


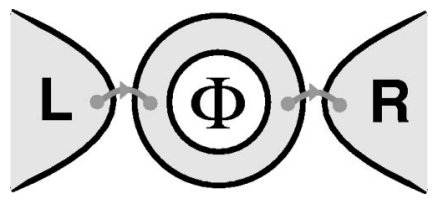

FIG. 4. The tunneling setup discussed in the text.

\section{AHARONOV-BOHM INTERFERENCE IN COTUNNELING THROUGH THE RING}

In the following, we will discuss the influence of the bath on the Aharonov-Bohm (AB) effect, i.e., on the flux dependence of the transport current through the ring. We consider tunneling into and out of the ring, taking place at two electrodes to the left and right of the ring (see Fig. 4). A tunneling situation is the appropriate one for our model, since attaching current leads would severely alter the system. Note that independent (sequential) tunneling only probes the density of states at the two contacts. Therefore, in order to observe the $\mathrm{AB}$ interference effect, we have to consider a Coulomb blockade situation, in which any electron tunneling into (or out of) the ring will enhance the total energy by the charging energy of the ring that is much larger than the bias voltage $V$ and the temperature $T$. In such a case, transport through the ring is possible only via cotunneling, ${ }^{21}$ i.e., a two-step process involving a virtual intermediate state belonging to a different number of electrons on the ring. A strong dependence of the tunneling current on the external magnetic flux, with a complete suppression at $\Phi_{0} / 2$ due to destructive interference, is visible only in the "elastic cotunneling" contribution, where the electronic state of the ring is left unaltered in the process. It is linear in the bias voltage and will dominate the inelastic contribution at low temperatures and for small bias voltages (see the discussion at the end of this section and Ref. 21).

Now consider cotunneling at $T=0$ under the influence of the bath. The semiclassical analysis of Aharonov-Bohm interference given in a preceding section is not applicable for $V \rightarrow 0$, since it assumes the electron can emit or absorb an arbitrary amount of energy. In the quantum-mechanical calculation, suppression of interference is due to the electron leaving a trace in the bath that permits, at least in principle, to decide which of the two arms of the ring the electron has traveled. This involves a transfer of energy between electron and bath. The bath spectrum determines the amount of bath oscillators able to absorb the small energy less than $e V$ that can be emitted by the electron. Therefore, it is to be expected that dephasing at zero temperature is suppressed for $V \rightarrow 0$ due to the energy conservation constraint. This will be confirmed by the calculation described in the following, although there are renormalization effects that change the strength of the tunneling current away from the point of perfect destructive interference, $\Phi=\Phi_{0} / 2$.

The tunneling process starts from a situation in which the ring is occupied by the equilibrium number of electrons (depending on the value of a gate voltage) and the Fermi seas in the left and right electrode are filled up to Fermi energies that differ by the bias voltage $e V$, see Fig. 5. Throughout the following discussion, we will assume the Fermi energy in the

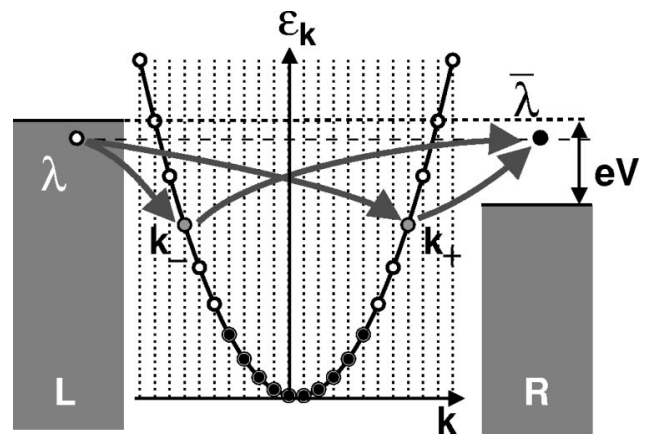

FIG. 5. Energy diagram for cotunneling through the $\mathrm{AB}$ ring at $\Phi=\Phi_{0} / 2$. The initial, final, and two possible intermediate states are indicated (see main text). The charging energy has to be added to the single-particle energy of the intermediate state shown here.

left electrode to be the larger one of the two (and $e V>0$ ). In the final state, an electron has appeared above the right Fermi sea, leaving behind a hole in the left electrode. Although we want to consider the situation where the electronic state of the ring has not changed in the end, the final state of the bath may be different. The intermediate state is characterized by an extra electron (or extra hole) present on the ring and some arbitrary state of the bath. Using standard second-order Fermi's Golden rule, the tunneling rate is obtained by summing over all intermediate states (dividing by the proper energy denominator) and all final states whose energy equals the initial energy,

$$
\Gamma=2 \pi \sum_{f}\left|\sum_{\nu} \frac{H_{f \nu}^{T} H_{\nu i}^{T}}{E_{\nu}-E_{i}}\right|^{2} \delta\left(E_{f}-E_{i}\right) .
$$

Here $\hat{H}^{T}=\hat{T}^{L}+\hat{T}^{R}$ is the sum of the tunneling Hamiltonians belonging to the left and the right junction, while the energies and eigenstates refer to the unperturbed Hamiltonian that includes everything besides tunneling. In particular, it includes the coupling between electrons and the bath, as well as the kinetic energies of electrons in the electrodes.

At this point we would like to emphasize that using Fermi's Golden rule for the calculation of the cotunneling current does not in itself mean taking into account the interaction between bath and system only in a perturbative way. The intermediate states being summed over in Eq. (48) are the exact eigenstates of the full system of electrons on the ring coupled to the fluctuating flux. In this sense, the coherence properties of the ring as a whole (including the bath) are tested by the cotunneling process. Applying the Golden rule in this context is roughly comparable to using the Kubo formula in a linear-response calculation of, e.g., the weaklocalization magnetoconductance, which does not automatically imply a perturbative description of the dephasing processes either. Still, there is an important difference. In our context, we essentially deal with a scattering situation, such that energy conservation holds at least for the initial and final states of the complete process. This will be seen to be important for our conclusions about the strength (or absence of) dephasing. What is neglected in our calculation are any equi- 
librium correlations between the state of the electrons in the leads and the state of the ring as a whole (including the fluctuating flux).

Before performing the calculation in the presence of the bath, we will briefly describe how the destructive interference at $\Phi_{0} / 2$ appears in this formula, in the situation without fluctuating flux. In such a case, the intermediate state $\nu$ refers solely to the electronic state $k$ on the ring, which is occupied by the additional electron in the course of tunneling. The final state $f$ is determined both by the state $\lambda$, which is unoccupied in the left electrode after the tunneling process, and the state $\bar{\lambda}$, where the electron ends up in the right electrode (see Fig. 5).

For simplicity, we will assume tunneling to take place only between two points, for example from a point $y_{L}$ at the tip of the left electrode to an adjacent point $x_{L}$ on the ring,

$$
\hat{T}^{L}=t_{L} \hat{\Psi}^{\dagger}\left(x_{L}\right) \hat{\Psi}\left(y_{L}\right)+\text { h.c. }
$$

and likewise for the right electrode. $t_{L}$ is a complex-valued tunneling amplitude. Such a description will be appropriate as long as the extent of the relevant region in which tunneling can take place is less than a wavelength.

The sum over intermediate electronic states $k$ on the ring then contains the following contribution that describes an electron going onto the ring from the left electrode and leaving through the right electrode,

$$
t_{L} t_{R}^{*} \Psi_{\lambda}\left(y_{L}\right) \Psi_{\lambda}^{*}\left(y_{R}\right) \sum_{k} \frac{\Psi_{k}\left(x_{R}\right) \Psi_{k}^{*}\left(x_{L}\right)}{\epsilon_{k}+E_{C}-\epsilon_{\lambda}} .
$$

Here the sum over $k$ is to be taken only over unoccupied single-electron states on the ring. $\Psi$ refers to single-electron wave functions on the ring and on the electrodes. Apart from the contribution listed here, there is another, completely analogous, contribution that belongs to the situation with an extra hole on the ring in the intermediate state. ${ }^{21}$ Note that for the purposes of our discussion we will not distinguish between the charging energies belonging to the electron and hole processes (assuming them to be of about the same magnitude).

Perfect destructive $\mathrm{AB}$ interference at an external static magnetic flux of $\Phi=\Phi_{0} / 2$ arises only for an even number of electrons on the ring. In this case, the energies

$$
\epsilon_{k}=\frac{1}{2 m}\left(k-\frac{2 \pi}{L} \frac{\Phi}{\Phi_{0}}\right)^{2}
$$

of the unoccupied states are pairwise degenerate, for $k_{+}$ $\equiv n 2 \pi / L$ and $k_{-} \equiv(1-n) 2 \pi / L$, see Fig. 5. Therefore, the energy denominators for $k_{+}$and $k_{-}$are the same, while the wave functions in the numerators produce a phase shift of $\exp \left[i\left(k_{+}-k_{-}\right) L / 2\right]=-1$ between the two possibilities, leading to complete cancellation of all terms in the sum. The same applies to the sum over occupied states (for the situation with an extra hole in the intermediate state).

Dephasing will, in general, "wash out" this perfect destructive interference. After taking the modulus squared of the sum of amplitudes given above in Eq. (50), which we

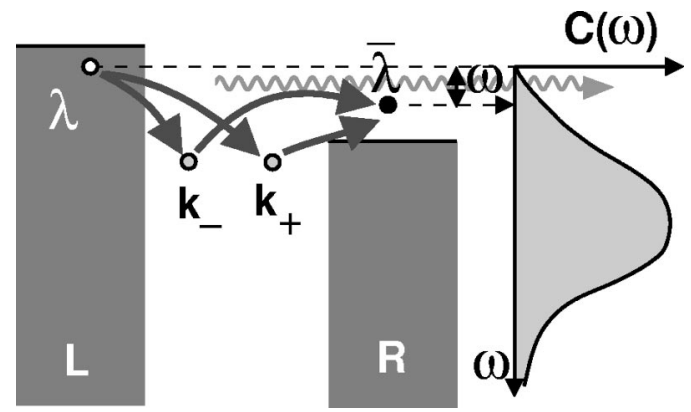

FIG. 6. Cotunneling in the presence of the fluctuating flux. Emission of a bath "phonon" of frequency $\omega$ leads to an incoherent contribution to the tunneling current, where the destructive interference between the two paths shown here is lost. The probability of emission depends on the bath spectrum $C(\omega)$.

briefly denote by $A(k)$, one obtains "classical" probabilities such as $\left|A\left(k_{+}\right)\right|^{2}$ but also cross terms of the form $A^{*}\left(k_{+}\right) A\left(k_{-}\right)$. A bath coupling to the electronic motion will affect these terms differently, if it is able to "distinguish" between the momenta $k_{+}$and $k_{-}$. Usually, the cross terms are suppressed. Then, the different contributions cannot cancel any more. Note that, away from perfect destructive interference, we have to expect an influence of the bath on the magnitude of the tunneling current under any circumstances, since mere renormalization effects such as a change in the effective mass of the electrons will be important. This is why we concentrate on the special case of $\Phi_{0} / 2$. Even in that situation, the interference minimum could vanish in a rather trivial way due to renormalization effects, if one chose a bath coupling asymmetrically to the two arms of the ring (or rather to left- and right-going momenta), thereby leading to different transmission probabilities. This is not the case in our model.

At this point, we can give a simple counting argument to arrive quickly at the voltage dependence of the "incoherent" contribution to the cotunneling rate $\Gamma$, which is produced by the fluctuating flux and leads to a nonvanishing current at $\Phi_{0} / 2$. The sum over initial electronic states on the left electrode is carried out over a region of extent $e V$. The probability of emission of a bath phonon is proportional to the bath spectrum $\omega^{\alpha}$, and we have to integrate this from 0 to the maximum energy of the electron, which is again of order $e V$. This yields a voltage dependence $\Gamma \propto V^{\alpha+2}$ of the incoherent contribution to the tunneling current (see Figs. 6 and 8). However, we want to analyze this in the following using a different, nonperturbative scheme, thereby making contact to the Feynman-Vernon influence functional formalism, ${ }^{4}$ which is the "workhorse" of many dephasing calculations.

To evaluate Eq. (48) in the presence of the bath, we rewrite the sum over intermediate states as a time integral

$$
\begin{aligned}
\sum_{\nu} \frac{H_{f \nu}^{T} H_{\nu i}^{T}}{E_{\nu}-E_{i}}= & i \int_{0}^{\infty} d t\left\langle f\left|\hat{H}^{T} \exp \left[-i\left(\hat{H}-E_{i}\right) t\right] \hat{H}^{T}\right| i\right\rangle \\
= & i \int_{0}^{\infty} d t \sum_{k} T_{\bar{\lambda} k}^{R} T_{k \lambda}^{L} \\
& \times\left\langle f^{B}\left|\exp \left[-i\left(\hat{H}[k]-E_{0}\right) t\right]\right| 0^{B}\right\rangle \\
& \times \exp \left[-i\left(E_{C}-\epsilon_{\lambda}\right) t\right] .
\end{aligned}
$$


In the second line, which replaces Eq. (50) in the presence of the bath, we have split off the contribution due to the electronic states. This has been possible because only the tunneling operators can change the electronic state, while the bath couples diagonally to the electronic-momentum eigenstates. Furthermore, we have confined ourselves to the process with an extra electron in the intermediate state, a completely analogous contribution for an extra hole has to be added. $\hat{H}[k]$ is the Hamiltonian for a given configuration consisting of an extra occupied state $k$ over the original Fermi sea on the ring. It only acts on the bath Hilbert space, where $0^{B}$ refers to the ground state of the bath prior to the tunneling event and $f^{B}$ is an arbitrary final state that the bath goes into after the cotunneling process is finished. In our notation, the sum of electronic kinetic energies is included in $\hat{H}[k]$ as well, whereas the charging energy $E_{C}$ has been taken into account separately. The matrix elements of the tunneling Hamiltonians $T^{L, R}$ are taken between the electronic states $\bar{\lambda}, k$, and $\lambda$ [compare Eqs. (49) and (50)]. $E_{0}$ is the ground-state energy of the ring, including the bath.

After taking the modulus squared of the sum given above, we arrive at the following contribution to the tunneling rate $\Gamma$ at zero temperature,

$$
\begin{aligned}
& 2 \pi \sum_{\lambda, \bar{\lambda}, k^{>}, k^{<}}\left(T_{\bar{\lambda} k^{>}}^{R} T_{k^{>} \lambda}^{L}\right)\left(T_{\bar{\lambda} k^{<}}^{R} T_{k^{<} \lambda}^{L}\right) * \int_{0}^{\infty} d \tau^{>} \exp \left[-i\left(E_{C}\right.\right. \\
& \left.\left.\quad-\epsilon_{\lambda}-E_{0}\right) \tau^{>}\right] \int_{0}^{\infty} d \tau^{<} \exp \left[+i\left(E_{C^{-}}-\epsilon_{\lambda}-E_{0}\right) \tau^{<}\right] \\
& \quad \times \sum_{f^{B}}\left\langle\chi^{<}\left(\tau^{<}\right) \mid f^{B}\right\rangle \delta\left[E_{f^{B}}-E_{0}-\left(\epsilon_{\lambda}-\epsilon_{\lambda}^{-}\right)\right] \\
& \quad \times\left\langle f^{B} \mid \chi^{>}\left(\tau^{>}\right)\right\rangle
\end{aligned}
$$

where $k^{>(<)}$denote unoccupied states on the ring. There are three analogous contributions besides the one shown here, in which the tunneling takes place in a different order (e.g., the process may start by an electron tunneling out of the ring, leaving a hole behind, etc.). Note that a similar expression arises in the derivation of the " $P(E)$ " theory of a tunnel junction coupled to a dissipative bath. ${ }^{22,23}$

In the preceding formula, the last line can be viewed as a kind of "generalized influence functional" $F\left[\tau^{>}, \tau^{<}, \omega=\epsilon_{\lambda}\right.$ $\left.-\epsilon_{\lambda}^{-}\right]$. It is equal to the overlap between bath states $\chi^{>(<)}$ that have been time evolved out of $0^{B}$ under the action of $\hat{H}\left[k^{>(<)}\right]$for some time $\tau^{>(<)}$. In contrast to the usual influence functional, the time of evolution may be different for the two states and the overlap is taken only with respect to bath states at an excitation energy $\omega$ (that must equal the energy emitted by the electron). This difference is due to the fact, that in our problem the energy-conservation constraint must be taken care of, since the electron cannot transfer an arbitrary amount of energy to the bath. This clearly shows why a single-particle calculation using the usual influence functional must fail when the amount of energy available is limited due to low temperatures or low bias voltages. This problem has also been discussed in Ref. 9, where the authors have used physical arguments to drop certain "zero-point"

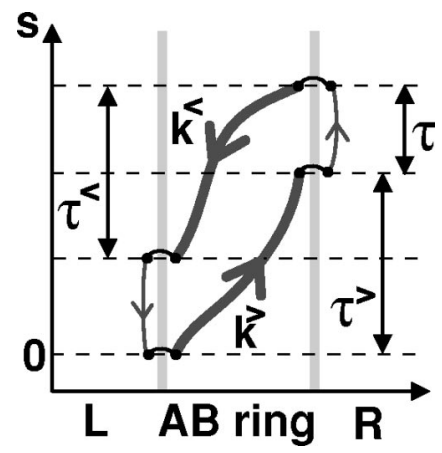

FIG. 7. Schematic "space-time" diagram showing the Keldysh contour that runs from 0 to $\tau$ and back again. The interaction operator that couples to the bath is nonvanishing only when there is an extra electron on the ring (thick lines), either in state $k^{>}$or $k^{<}$(see main text). Tunneling processes are indicated.

contributions to the dephasing rate obtained in a singleparticle calculation. The normal influence functional is recovered by integrating over all possible energy transfers and setting $\tau^{>}=\tau^{<}$,

$$
F[\tau]=\left\langle\chi^{<}(\tau) \mid \chi^{>}(\tau)\right\rangle=\int_{-\infty}^{+\infty} d \omega F[\tau, \tau, \omega]
$$

If no bath is present or its spectrum has a lower cutoff that is larger than the energy available to the electron, energy conservation leads to $\omega \equiv 0$, such that the final and initial bath states coincide, $f^{B} \equiv 0^{B}$. Then, $F$ is a product of a factor depending only on $k^{>}$and another one, depending only on $k^{<}$. In this case, the sums over $k^{>(<)}$may be carried out separately, like before, and the terms will cancel again (for $\Phi_{0} / 2$ ), provided the bath couples equally to $k_{+}$and $k_{-}$(see discussion above). Although there is definitely no dephasing in this case, the magnitude of the tunneling current may be changed for $\Phi \neq \Phi_{0} / 2$, due to the afore-mentioned renormalization effects.

The Fourier transform (in $\omega$ ) of the generalized influence functional may be written as follows:

$$
F\left[\tau^{>}, \tau^{<}, \tau\right]=\frac{e^{+i E_{0} \tau}}{2 \pi}\left\langle\chi^{<}\left(\tau^{<}\right)\left|e^{-i \hat{H}[0] \tau}\right| \chi^{>}\left(\tau^{>}\right)\right\rangle .
$$

It can be represented as a Keldysh time-ordered expectation value, ${ }^{24,25}$ apart from a prefactor $\exp \left[-i E_{0}\left(\tau^{>}-\tau^{<}\right)\right]$,

$$
\left\langle\hat{T}_{K} \exp \left[-i \oint_{K} d s \hat{V}_{I}(s)\right]\right\rangle_{0^{B}} .
$$

Here, $\quad \hat{V}_{I}(s) \equiv g \tilde{k} \hat{\phi}(s) / m+\widetilde{k}^{2} /(2 m), \quad$ with $\quad \widetilde{k} \equiv k-2 \pi \Phi /$ $\left(\Phi_{0} L\right)$, where $\Phi$ is the additional static flux. $\hat{V}_{I}$ couples the additional electron in state $k$ to the bath (and also incorporates the kinetic energy). We have $k=k^{>}$if $s$ is on the forward time branch and $0 \leqslant s \leqslant \tau^{>}$, while $k=k^{<}$, if $s$ is on the backward time branch and $\tau+\tau^{>}-\tau^{<} \leqslant s \leqslant \tau+\tau^{>}$. For all other times, $\hat{V}_{I}$ vanishes. This is represented graphically in Fig. 7. Note that $\hat{V}_{I}$ is taken in the interaction picture with respect to the bath coupled to the original Fermi sea on the 
ring. Once more, we have neglected the term $g^{2} \hat{\Phi}^{2}$ that turns out to be unimportant for the bath spectra considered below.

Using Keldysh time-ordering and a linked-cluster expansion (Wick's theorem), we can represent Eq. (56) as an exponential containing double time integrals involving the Keldysh time-ordered correlation function of the bath operator $\hat{\phi}$,

$$
\begin{aligned}
\exp \left[-i \oint_{K} d s\left\langle\hat{V}_{I}(s)\right\rangle\right] \\
\times \exp \left[-\frac{1}{2} \oint_{K} d s_{1} \oint_{K} d s_{2}\left\langle\hat{T}_{K} \delta \hat{V}_{I}\left(s_{1}\right) \delta \hat{V}_{I}\left(s_{2}\right)\right\rangle\right] .
\end{aligned}
$$

We have set $\delta \hat{V}_{I} \equiv \hat{V}_{I}-\left\langle\hat{V}_{I}\right\rangle=g \widetilde{k} \hat{\phi} / m$. The principal steps involved in the evaluation of this expression are demonstrated in the Appendix, where we show how the usual Caldeira-Leggett influence functional for a bath of harmonic oscillators can be derived very efficiently using this method. The resulting exponential couples the momenta $k^{>}$and $k^{<}$ and may therefore lead to dephasing.

From now on, we again consider baths that are characterized by a power-law spectrum at low frequencies, $C(\omega)$ $\propto \omega^{\alpha}$ with an exponent $\alpha \geqslant 1$. Remember that the case $\alpha$ $=1$ represents fluctuations of the magnetic flux produced by Nyquist noise of an external current loop. For these bath spectra, it is sufficient to carry out an expansion of the cotunneling rate to leading order in the coupling strength $g$. The part of the resulting expression that couples $k^{>}$and $k^{<}$ is seen to lead, after summation over all electronic states $k^{>}$, $k^{<}, \lambda, \bar{\lambda}$, to an "incoherent" contribution that washes out destructive interference but that is suppressed for low-bias voltages, as expected.

At zero temperature, the ratio of this incoherent current at $\Phi=\Phi_{0} / 2$ to the normal elastic cotunneling current that flows at $\Phi=0$ is found to be given by the following approximate expression (up to a constant of order 1),

$$
\left[g^{2} v_{F}^{2} \int_{0}^{e V}\left(1-\frac{\omega}{e V}\right) C(\omega) d \omega\right] \frac{1}{\delta \epsilon^{2}}
$$

The expression inside the brackets can be interpreted as the variance of the fluctuating energy of a single-particle level on the ring. However, it is to be evaluated taking into account only the fluctuations up to the frequency corresponding to the bias voltage and using a weight factor $1-\omega / \mathrm{eV}$ that favors low-energy transfers $\omega$. We have already pointed out that the cutoff at $e V$ is a simple consequence of energy conservation. For a power-law bath spectrum $C(\omega) \propto \omega^{\alpha}$, the integral yields a voltage dependence proportional to $V^{\alpha+1}$, so the incoherent tunneling current goes as $V^{\alpha+2}$. Note that $\delta \epsilon=h v_{F} / L$ refers to the single-particle level spacing on the ring. The qualitative behavior of the cotunneling rate as a function of both external static flux and bias voltage is shown in Fig. 8. (We remark again that the incoherent current due to external Nyquist noise would be too small to be

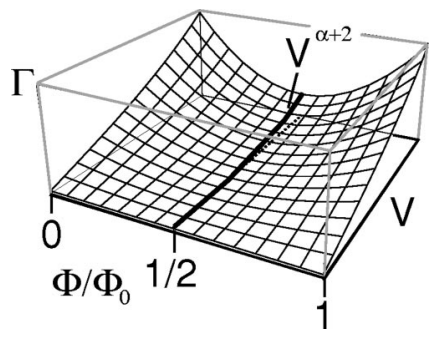

FIG. 8. Schematic behavior of the cotunneling rate as a function of static magnetic flux and bias voltage. In the ideal situation, the rate vanishes at $\Phi_{0} / 2$ (dotted line), while it rises as a power of the bias voltage due to the incoherent contribution resulting from the fluctuations of the flux (thick line).

actually measurable under reasonable experimental conditions, compare the discussion at the end of Sec. IV D).

Thus, we see that suppression of destructive interference does not show up in the linear conductance. In this sense, the fluctuations do not lead to dephasing in the linear-transport regime at zero temperature. For the Nyquist case $\alpha=1$, the exponent of $V$ is the same as that for inelastic electronic cotunneling processes in a system with a continuum of intermediate electronic states. ${ }^{21}$ Bath spectra with $\alpha>1$ obviously lead to an even weaker decrease in the visibility of the interference minimum at low-bias voltages. Note that formally inserting $\alpha=-1$ in $\Gamma \propto V^{\alpha+2}$ would lead to an incoherent contribution to the linear conductance even at $T=0$. However, this case is not of interest here, since it cannot be produced by a fluctuating magnetic flux and it is not covered by the approximations made in our calculation (in particular dropping the $\hat{\phi}^{2}$ term). It would correspond to the strong force fluctuations of an Ohmic Caldeira-Leggett bath used in the description of quantum Brownian motion.

For bias voltages $\mathrm{eV}$ smaller than the single-particle energy spacing $\delta \epsilon$ on the ring, dephasing is merely due to the coupling to the fluctuating flux. At higher voltages, the inelastic cotunneling processes become important. In these, one electron tunnels into the ring, while another electron goes out at the opposite electrode, thus leaving behind a particle-hole excitation on the ring. ${ }^{21}$ Since all the corresponding final states are different, their contributions to the cotunneling current sum up incoherently. Therefore, similar to dephasing produced by the bath, they also lead to a nonvanishing contribution to the tunneling current at $\Phi_{0} / 2$, where, ideally, one should have perfect destructive interference. The number of possibilities to create a particle-hole excitation with an energy of at most $\mathrm{eV}$ is $\propto(e V / \delta \epsilon)^{2}$, if we assume $e V \gg \delta \epsilon$. In that regime, the ratio of the incoherent current contribution due to the external bath to the electronic inelastic contribution is given by the bracket in Eq. (58), multiplied by $\left[S \epsilon /\left(E_{c} e V\right)\right]^{2}$ The electronic inelastic contribution will be the dominant one.

Finally, let us discuss finite temperatures. Without the bath and as long as $T \ll \delta \epsilon$, only the Fermi distributions in the electrodes get smeared, which does not affect the tunneling current, if one takes into account that now the tunneling processes do not only lead to an electron transport from left to right but in the other direction as well. The presence of the 
bath will introduce some temperature dependence for the incoherent current contribution in this regime, since at finite temperatures the tunneling electron will not only emit an energy quantum into the bath but also absorb a thermal-bath excitation. Therefore, the energy $\omega$ transferred to the bath now can be negative as well. There is no restriction on the amount of energy an electron can absorb, so there is no cutoff $e V$ for negative $\omega$. At positive energy transfers, the probability of spontaneous emission into the bath $[\propto C(\omega)$ in Eq. (58)] now has to be multiplied by $n(\omega)+1$, where $n(\omega)$ is the Bose distribution function (induced emission). At negative $\omega$, this is replaced by $n(|\omega|)$, since only absorption of thermal excitations (not of vacuum fluctuations) is possible.

Taking into account the thermal smearing of the electrode Fermi distributions, the balance of left and right-going tunneling currents and the induced emission/absorption mentioned just before, we have to perform the following replacements in Eq. (58),

$$
\begin{aligned}
\int_{0}^{e V} d \omega\left(1-\frac{\omega}{e V}\right) C(\omega) \mapsto & \int_{-\infty}^{+\infty} d \omega W(\beta, \omega, e V)(n(|\omega|) \\
& +\theta(\omega)) C(|\omega|)
\end{aligned}
$$

Note that the factor multiplying $W$ in the integral corresponds directly to the function $P(E)$ that occurs in the theory of tunneling in a dissipative environment. ${ }^{22,23}$ The function $W$ itself represents an integral over the average of energies in the left and right electrodes, $\epsilon \equiv\left(\epsilon_{\lambda}+\epsilon_{\lambda}^{-}\right) / 2$, at a fixed energy transfer $\omega \equiv \epsilon_{\lambda}-\epsilon_{\bar{\lambda}}$,

$$
W(\beta, \omega, e V) \equiv(e V)^{-1} \int d \epsilon f_{L}\left(1-f_{R}\right)-f_{R}\left(1-f_{L}\right) e^{-\beta \omega}
$$

Here $f_{L}=f_{\beta}\left(\epsilon_{\lambda}-e V / 2\right)$ and $f_{R}=f_{\beta}\left(\epsilon_{\lambda}^{-}+e V / 2\right)$ are the Fermi distributions in the two electrodes. The factor of $\exp (-\beta \omega)$ is due to the fact that for an electron going from right to left the energy transferred to the bath is $-\omega$, so the ratio between the values of $n(|\omega|)+\theta(\omega)$ at positive and negative frequencies appears, which is just this factor. Note that, in the low-temperature limit $(\beta \rightarrow \infty)$, the function $W$ becomes $1-\omega / e V$ for $\omega<e V$ and vanishes for $\omega>e V$. This reproduces the left-hand side of Eq. (59) as a special case.

Using this formula, the incoherent tunneling current is found to be enhanced by a temperature-dependent contribution proportional to $T^{\alpha+1} V$.

At $T \geqslant \delta \epsilon$, one would have to take into account the thermal averaging over different electronic configurations on the ring (still at a fixed particle number determined by charging energy and gate voltage). The perfect destructive interference at $\Phi_{0} / 2$ depends on the presence of an electronic configuration that is symmetric in the occupancy of equal-energy states having $k>0$ and $k<0$ (see discussion above). The thermal average includes other configurations as well and therefore leads to a suppression of the destructive interference in the elastic tunneling current, even without the bath.
Furthermore, the electronic inelastic contribution is also enhanced at finite temperatures and becomes linear in the voltage. $^{21}$

\section{DISCUSSION}

Since we do not find dephasing in our model at zero temperature (under the circumstances specified above), the reader may wonder how our result is related to some apparently contradicting conclusions in the literature. First of all, we would like to point out that a comparison between findings in different physical situations is not straightforward, since there is no general definition of a "dephasing rate." E.g., in recent work on an Aharonov-Bohm ring containing a quantum dot capacitively coupled to the Ohmic fluctuations in a gate,${ }^{10}$ the authors found that the coupling suppresses the magnitude of the persistent current flowing in the ring and induces fluctuations of this current. The effect persists even at $T=0$ and was interpreted as dephasing at zero temperature. Formally, the coupling assumed in the setup of Ref. 10 is nondiagonal in the system's eigenbasis, whereas it is diagonal in our case. This may indeed lead to a weaker tendency towards dephasing in our model (see the discussion at the end of Sec. II). Still, we also find both a reduction of the persistent current (Sec. IV A) and fluctuations (caused by the vector-potential fluctuations, since the momentum is conserved), regardless of the details of the bath spectrum. Nevertheless, this apparently does not imply dephasing in our interference setup (cotunneling transport situation), which has been our main concern here and that has no analogy in the work of Ref. 10. The transport effect depends in an essential way on the long-time dynamics of the system and, therefore, on the low-frequency behavior of the bath spectrum (similarly to the Caldeira-Leggett model or some aspects of the spin-boson model), while the reduction of the persistent current does not. Therefore, we have not interpreted the reduction of the persistent current in terms of zerotemperature dephasing, although it certainly constitutes a suppression of an interference-related phenomenon. We believe that the suppression of interference in a cotunneling setup considered here may be more directly related to mesoscopic transport interference experiments.

Our model is certainly quite removed from the discussions about low-temperature dephasing in weak localization. There, dephasing by electron-electron interaction in an extended disordered system weakens the coherent backscattering effect, which is an interference phenomenon that is robust against both thermal and impurity averaging, in contrast to the destructive interference considered in our model. Since the electrons inside the metal interact all the time, it is not obvious whether that situation is in any way analogous to the kind of "scattering" situation considered here for the cotunneling transport.

\section{CONCLUSIONS}

We have analyzed a simple model of a fluctuating magnetic flux threading an Aharonov-Bohm ring and discussed its effects on equilibrium properties, such as persistent cur- 
rent and tunneling density of states, as well as transport properties such as the two-particle Green's function and the cotunneling current through the ring. Particular emphasis has been put on the question of dephasing and low-temperature behavior. There are important qualitative differences depending on the low-frequency behavior of the bath power spectrum. For the case of Nyquist noise and an arbitrary initial superposition of momentum states, an exponential decay of off-diagonal elements of the density matrix in time at $T>0$ goes over into "power-law dephasing" at zero temperature. However, if one probes the coherence properties of the electronic motion on the ring by checking for the possible suppression of destructive $\mathrm{AB}$ interference in a cotunneling setup, no such suppression is found in the linear-transport regime at $T=0$. This is because the possibility for the electron to leave a trace in the bath is diminished due to the energy-conservation constraint. Our calculation serves as an illustrative example of the difference between the "optics" type of interference experiments (employing single particles), in which the semiclassical approximation and/or the usual Feynman-Vernon influence functional may be applied to discuss dephasing, and the linear-transport interference experiments encountered in mesoscopic physics, in which special care has to be taken in the analysis of dephasing at low temperatures.

\section{ACKNOWLEDGMENTS}

We would like to acknowledge valuable discussions with M. Büttiker, Y. Imry, G.-L. Ingold, A. J. Leggett, D. Loss, Yu. V. Nazarov, A. Rosch, E. Sukhorukov, A. Zaikin, and W. Zwerger. Our work is supported by the Swiss NSF.

\section{APPENDIX: DERIVATION OF CALDEIRA-LEGGETT INFLUENCE FUNCTIONAL USING KELDYSH TIME-ORDERING AND WICK'S THEOREM}

The influence functional for a system (variable $\hat{x}$ ) coupled linearly to a linear bath $(\hat{\phi})$ is usually derived in the pathintegral picture, by "integrating out" the bath variables. ${ }^{1,2,4}$ This can be done because the bath consists of a set of uncoupled harmonic oscillators. However, the calculation is usually quite cumbersome, although the result is simple enough and involves only the real and imaginary parts of the bath correlator $\left\langle\hat{\phi}\left(t_{1}\right) \hat{\phi}\left(t_{2}\right)\right\rangle$. Here we present a derivation based on Keldysh time-ordering and Wick's theorem. The oscillators of the bath and their action for an external driving force never have to be considered and therefore this is probably the shortest route to the well-known Caldeira-Leggett influence functional. Modifications of this approach are applicable in more complicated situations as well (compare the main text).

The Feynman-Vernon influence functional is the overlap between the two bath states that result from the action of two different (fixed) system trajectories $x^{>}(\cdot)$ and $x^{<}(\cdot)$ onto the same initial bath state $\chi_{0}$. A thermal average over $\chi_{0}$ has to be performed at finite temperatures,

$$
J\left(x^{<}, x^{>}\right)=\left\langle U\left(t, 0 \mid x^{<}\right) \chi_{0} \mid U\left(t, 0 \mid x^{>}\right) \chi_{0}\right\rangle_{\chi_{0}} .
$$

Here the time-evolution operators depend on the system trajectory $x(\cdot)$ via the interaction term $\hat{V}(t)=x(t) \hat{\phi}$ in the Hamiltonian. Using the interaction picture with respect to the bath Hamiltonian $\hat{H}_{B}$, we can explicitly write down $J$ in the following form, with (anti-)time-ordering symbols $\hat{T}(\tilde{\hat{T}})$,

$$
\begin{aligned}
J= & Z_{B}^{-1} \operatorname{tr}\left[e^{-\beta \hat{H}_{B}} \tilde{\hat{T}} \exp \left[+i \int_{0}^{t} x^{<}(s) \hat{\phi}(s) d s\right]\right. \\
& \left.\times \hat{T} \exp \left[-i \int_{0}^{t} x^{>}(s) \hat{\phi}(s) d s\right]\right] .
\end{aligned}
$$

Here $Z_{B} \equiv \operatorname{tr} \exp \left(-\beta \hat{H}_{B}\right)$. Now we can use Keldysh time ordering to abbreviate this formally,

$$
J=Z_{B}^{-1} \operatorname{tr}\left[e^{-\beta \hat{H}_{B}} \hat{T}_{K} \exp \left[-i \oint_{K} x_{K}(s) \hat{\phi}(s) d s\right]\right]
$$

Here $x_{K}(s)$ is equal to $x^{>}(s)$ [or $x^{<}(s)$ ] if $s$ lies on the forward (or backward) part of the Keldysh contour that runs from 0 to $t$ and back again. The advantage of this formal rearrangement is that the application of Wick's theorem (leading to a linked-cluster expansion) now becomes very simple. We immediately obtain for $J$,

$$
\exp \left[-\frac{1}{2} \oint_{K} d t_{1} \oint_{K} d t_{2}\left\langle\hat{T}_{K} \hat{\phi}\left(t_{1}\right) \hat{\phi}\left(t_{2}\right)\right\rangle x_{K}\left(t_{1}\right) x_{K}\left(t_{2}\right)\right] .
$$

The brackets $\langle\cdot\rangle$ denote the thermal average with respect to $\hat{H}_{B}$. Now we can translate back the exponent by taking into account all four possible combinations of the two times on the forward or backward time paths: (To keep the notation short, we use subscripts for the time arguments)

$$
\begin{aligned}
& -\frac{1}{2} \int_{0}^{t} d t_{1} \int_{0}^{t} d t_{2}\left\{\left\langle\hat{T} \hat{\phi}_{1} \hat{\phi}_{2}\right\rangle x_{1}^{>} x_{2}^{>}+\left\langle\tilde{T} \hat{\phi}_{1} \hat{\phi}_{2}\right\rangle x_{1}^{<} x_{2}^{<}\right. \\
& \left.-\left\langle\hat{\phi}_{1} \hat{\phi}_{2}\right\rangle x_{1}^{<} x_{2}^{>}-\left\langle\hat{\phi}_{2} \hat{\phi}_{1}\right\rangle x_{1}^{>} x_{2}^{<}\right\} .
\end{aligned}
$$

This can be simplified further by noting that the real part of all the four different correlators appearing here is the same, since it is symmetric in the time arguments: $2 \operatorname{Re}\left\langle\hat{\phi}_{1} \hat{\phi}_{2}\right\rangle=\left\langle\left\{\hat{\phi}_{1}, \hat{\phi}_{2}\right\}\right\rangle$. Therefore, the real part of the exponent is given by:

$$
-\frac{1}{2} \int_{0}^{t} d t_{1} \int_{0}^{t} d t_{2} \operatorname{Re}\left\langle\hat{\phi}_{1} \hat{\phi}_{2}\right\rangle\left(x_{1}^{>}-x_{1}^{<}\right)\left(x_{2}^{>}-x_{2}^{<}\right) .
$$

It defines the imaginary part of the influence action $S_{\text {infl }}\left[x^{>}, x^{<}\right]$and describes dephasing and heating due to the fluctuations of the bath variable $\hat{\phi}$.

Treating the imaginary part is only slightly more difficult. We have 


$$
\begin{gathered}
\operatorname{Im}\left\langle\hat{\phi}_{1} \hat{\phi}_{2}\right\rangle=-\operatorname{Im}\left\langle\hat{\phi}_{2} \hat{\phi}_{1}\right\rangle=\frac{1}{2 i}\left\langle\left[\hat{\phi}_{1}, \hat{\phi}_{2}\right]\right\rangle \\
\operatorname{Im}\left\langle\hat{T} \hat{\phi}_{1} \hat{\phi}_{2}\right\rangle=\operatorname{sgn}\left(t_{1}-t_{2}\right) \operatorname{Im}\left\langle\hat{\phi}_{1} \hat{\phi}_{2}\right\rangle=-\operatorname{Im}\left\langle\widetilde{T} \hat{\phi}_{1} \hat{\phi}_{2}\right\rangle .
\end{gathered}
$$

In order to get rid of the $\operatorname{sgn}\left(t_{1}-t_{2}\right)$, we split the double time integral into one part where $t_{2}<t_{1}$ and one with $t_{2}$ $>t_{1}$. In the latter part, we interchange integration variables $t_{1}$ and $t_{2}$. Then we obtain for the imaginary part of the exponent:

$$
-i \int_{0}^{t} d t_{1} \int_{0}^{t_{1}} d t_{2} \operatorname{Im}\left\langle\hat{\phi}_{1} \hat{\phi}_{2}\right\rangle\left(x_{1}^{>}-x_{1}^{<}\right)\left(x_{2}^{>}+x_{2}^{<}\right) .
$$

This defines the real part of the influence action and describes friction and renormalization effects (e.g., effective mass). Note that we can bring the real part of the exponent, (A6), to a similar form by cutting off the $t_{2}$-integral at $t_{1}$ and dropping the factor $1 / 2$ in front of the expression, since there the integrand is symmetric in $t_{1}, t_{2}$.

In this way we have arrived at the well-known influence functional for a system coupled linearly to a linear bath. As usual, a coupling of the form $f(x) \hat{\phi}$ just leads to a replacement $x \mapsto f(x)$ in the final expression, and something such as $\sum_{j} f_{j}(x) \hat{\phi}_{j}$ leads to a sum of the corresponding influence actions, if the $\hat{\phi}_{j}$ are uncorrelated.
${ }^{1}$ A.O. Caldeira and A.J. Leggett, Ann. Phys. (N.Y.) 149, 374 (1983); Physica A 121, 587 (1983).

${ }^{2}$ U. Weiss, Quantum Dissipative Systems (World Scientific, Singapore, 2000).

${ }^{3}$ C. Park and Y. Fu, Phys. Lett. A 161, 381 (1992).

${ }^{4}$ R.P. Feynman and F.L. Vernon, Ann. Phys. (N.Y.) 24, 118 (1963).

${ }^{5}$ D. Loss and T. Martin, Ann. Phys. (N.Y.) 47, 4619 (1993).

${ }^{6}$ D. Loss and K. Mullen, Phys. Rev. B 43, 13252 (1991).

${ }^{7}$ A. Stern, Y. Aharonov, and Y. Imry, Phys. Rev. A 41, 3436 (1990).

${ }^{8}$ See P. Mohanty, E.M.Q. Jariwala, and R.A. Webb, Phys. Rev. Lett. 78, 3366 (1997) for recent experiments; D.S. Golubev and A.D. Zaikin, ibid. 81, 1074 (1998); Physica B 255, 164 (1998); Phys. Rev. B 59, 9195 (1999); 62, 14061 (2000) for the claim that there is dephasing even at $T=0$; I.L. Aleiner, B.L. Altshuler, and M.E. Gershenson, Phys. Rev. Lett. 82, 3190 (1999); Waves Random Media 9, 201 (1999) for a criticism of this claim.

${ }^{9}$ D. Cohen and Y. Imry, Phys. Rev. B 59, 11143 (1999).

${ }^{10}$ P. Cedraschi, V.V. Ponomarenko, and M. Büttiker, Phys. Rev. Lett. 84, 346 (2000).

${ }^{11}$ P. Cedraschi and M. Büttiker, Phys. Rev. B 63, 165312 (2001); Ann. Phys. (N.Y.) 289, 1 (2001).

${ }^{12}$ M. Büttiker, cond-mat/0106149 (unpublished).
${ }^{13}$ V.E. Kravtsov and B.L. Altshuler, Phys. Rev. Lett. 84, 3394 (2000).

${ }^{14}$ P.A. Mello, Y. Imry, and B. Shapiro, Phys. Rev. B 61, 16570 (2000).

${ }^{15}$ G. Seelig and M. Büttiker, cond-mat/0106100 (unpublished).

${ }^{16}$ G.W. Ford, J.T. Lewis, and R.F. O'Connell, Phys. Rev. A 37, 4419 (1988).

${ }^{17}$ V. Hakim and V. Ambegaokar, Phys. Rev. A 32, 423 (1985).

${ }^{18}$ P. Schramm and H. Grabert, J. Stat. Phys. 49, 767 (1987).

${ }^{19}$ T.A. Welton, Phys. Rev. 74, 1157 (1948).

${ }^{20}$ P.W. Milonni, The Quantum Vacuum (Academic Press, San Diego, 1994).

${ }^{21}$ D.V. Averin and Yu.V. Nazarov, in Single Charge Tunneling, edited by H. Grabert and M. H. Devoret (Plenum Press, New York, 1992).

${ }^{22}$ G.-L. Ingold and Yu.V. Nazarov, in Single Charge Tunneling (Ref. 21).

${ }^{23}$ G. Schön, in Quantum Transport and Dissipation, edited by T. Dittrich et al. (Wiley-VCH, Weinheim, 1998).

${ }^{24}$ L.V. Keldysh, Zh. Éksp. Teor. Fiz. 47, 1515 (1964) [Sov. Phys. JETP 20, 1018 (1965)].

${ }^{25}$ A. Zagoskin, Quantum Theory of Many-Body Systems (Springer, New York, 1998). 